\title{
Parental Leave and Mothers' Careers: The Relative Importance of Job Protection and Cash Benefits
}

\author{
RAFAEL LALIVE \\ University of Lausanne and CEPR \\ ANALÍA SCHLOSSER \\ Tel Aviv University \\ ANDREAS STEINHAUER \\ University of Zurich \\ and \\ JOSEF ZWEIMÜLLER \\ University of Zurich and CEPR
}

First version received June 2011; final version accepted February 2013 (Eds.)

\begin{abstract}
Job protection and cash benefits are key elements of parental leave (PL) systems. We study how these two policy instruments affect return-to-work and medium-run labour market outcomes of mothers of newborn children. Analysing a series of major PL policy changes in Austria, we find that longer cash benefits lead to a significant delay in return-to-work, particularly so in the period that is job-protected. Prolonged parental leave absence induced by these policy changes does not appear to hurt mothers' labour market outcomes in the medium run. We build a non-stationary model of job search after childbirth to isolate the role of the two policy instruments. The model matches return-to-work and return to same employer profiles under the various factual policy configurations. Counterfactual policy simulations indicate that a system that combines cash with protection dominates other systems in generating time for care immediately after birth while maintaining mothers' medium-run labour market attachment.
\end{abstract}

Key words: Parental leave, family and work obligations, return-to-work, labour supply, earnings, family earnings gap

JEL Codes: J13, J18, J22

\section{INTRODUCTION}

Parental leave $(P L)$ regulations are a central element of family policies in most OECD countries. They help new parents in two complementary ways: by guaranteeing the pre-birth job and by offering financial support. A first main goal of PL mandates is to increase women's employment and earnings in the medium run by encouraging job continuity after birth. Yet, prolonged periods of absence from the workplace might lead to loss of human capital and weaker labour market prospects after returning to work. Hence previous employers, while obliged to re-employ mothers 
after the baby break, may either remunerate them worse than their colleagues or may dismiss them with a higher probability when job protection has run out. Moreover, longer workplace absences after a birth may lead to lower pay and less stable employment for women that move to new employers.

A second main goal of PL provisions is to mitigate financial hardships associated with the increase in family size and with foregone earnings when the mother needs to stay home with the newborn child. In terms of labour market outcomes, cash benefits are expected to decrease incentives to return-to-work inducing women to return later after the baby break and worsen medium-run labour market outcomes. However, a longer duration of benefits payments might allow mothers to search for better jobs during the leave improving job stability and earnings. In addition, a longer period shared between mother and child during the first months after a birth might benefit health of the child and of the mother fostering her medium-run labour market performance 1

A PL system that generates time for care without sacrificing labour market attachment of mothers of newborn children is attractive in a setting where mothers value time with their child very strongly right after birth and child care is expensive. Actual PL systems differ strongly across countries. Some countries offer very short leaves without any benefits (like the 1993 Family and Medical Leave Act in the U.S.) whereas other countries offer long leaves associated with government-financed cash benefits (like Germany and France). Despite the widespread prevalence of PL policies and the huge cross-country differences of PL systems, their impact on women's labour market performance is not well understood. In particular, not much is known about the (isolated and interaction) effects of the two main policy instruments: the maximum duration of job protection and the maximum duration of cash benefits.

The aim of this article is to shed light on the effects of these two policy parameters. Our aim is 2-fold. First, we estimate the causal impact of alternative PL systems (in terms of cash benefits and job protection) on return-to-work behaviour and labour market outcomes in the medium-run in Austria. Austria provides an attractive experimental environment to study the effects of PL policies. Thanks to the almost universal eligibility of parental leave among working women and the high take-up rates, differences in access or selection problems due to differential take-up are not a concern in our set-up. Moreover, the high eligibility rates combined with the high take-up rates allow us to generate estimates for the causal effects of PL that approach treatment effects for the whole Austrian population. In addition changes to PL rules affect a large variety of women to a large extent given the high eligibility and take-up rates. Finally, the Austrian environment is appealing because PL policy changes were substantial (maximum parental leave durations varied between 1 year and 2.5 years). The various policy regimes in Austria cover a large range of PL durations that are observed in a cross-section of OECD countries.

Austria modified key aspects to its PL system during the 1990s. These successive policy changes allow us to identify the causal effect of alternative PL systems on short- and medium-run labour market outcomes. To empirically identify the causal effects of alternative PL regimes, we exploit variation in PL regulations generated by three policy changes that took place in Austria during the 1990s. The first policy change, implemented on July 1, 1990, extended the maximum duration of both cash benefits and job protection from the child's first to the child's second birthday. The second policy change, implemented on July 1, 1996, reduced the maximum duration of cash

1. Australia introduced a PL system as of January 1, 2011. The directive that introduces the system explicitly states that "The objective of the scheme is to provide financial support to primary carers (mostly birth mothers) in order to allow those carers to take time off work to care for the child after the child's birth, and encourage women to continue to participate in the workforce [...]." (Paid Parental Leave Guide Article 1.2.1.) This shows that PL aims to provide time for care without jeopardizing medium run labour force attachment. 
benefits to the date when the child turns 18 months old, keeping job protection unchanged. The third policy change, implemented on July 1, 2000, increased the maximum duration of cash benefits to the date when the child turns 30 months old, again keeping job protection unchanged. This setting is interesting because we can estimate the effects of (i) a system where cash benefits and job protection last equally long (but their maximum length varies over time); (ii) a system where cash benefits last shorter than job protection (so that part of the job protected leave is unpaid); and (iii) a system where cash benefits last longer than job protection (so that part of the leave is paid but not job protected).

Each of the three policy changes was implemented on July 1 of the respective year. This yields a simple but powerful empirical research design that allows us to compare return-to-work behaviour and labour market outcomes of mothers who gave birth in July or August to mothers who gave birth in May or June. A major advantage of this design is that endogenous selection into treatment and comparison groups is quite unlikely. This is because the policy changes could not be anticipated: the children were already conceived when the changes were announced. Moreover, to rule out that our estimates are driven by seasonality we include, as an additional comparison group, mothers who gave birth between May and August of the years preceding the policy changes. Hence our econometric analysis combines a regression discontinuity design with a difference-in-differences approach.

Our empirical analysis relies on a large and informative data set, the Austrian Social Security Database (ASSD), which covers the universe of Austrian employees and contains information on individuals' earnings and employment histories and take-up of government transfers. We are able to examine mothers' employment and earnings starting 2 years before they gave birth and track their post-birth labour market outcomes for a significant number of years.

Our main findings from the empirical analysis can be summarized as follows. Longer PL durations significantly delay return-to-work. Extending PL benefits and job protection by one year (the 1990 reform) increases time at home by 7.8 months. Reducing the duration of benefit payments by 6 months while keeping job protection at 24 months (the 1996 reform) shortens time at home by 3.4 months. Finally, extending payment duration by 12 months (to 30 months) while guaranteeing job protection for only 24 months (the 2000 reform) extends time at home by 3 months. Nevertheless, despite the significant delays in return-to-work among mothers exposed to the more generous leave regimes, we do not find any detrimental effects on their labour market outcomes in the medium-run.

Yet, information on these policy regimes does not allow us to fully disentangle the role of benefits and job protection since Austria always used both. This is why, in a second step, we set up a non-stationary model of job search after birth in the context of a PL system that offers cash and job protection. We consider the decision of a mother who, after interrupting work due to the birth of a child, decides optimally when to return-to-work and whether or not to return to the same employer or to a new employer. Women get job offers from new employers on a continuous basis and have a time-delimited option to go back to the same employer. When a sufficiently attractive job offer from a new employer arrives, women stop the baby break and return to work. Our model features heterogeneity both in wages and in the value that mothers attach to time at home (home production). Moreover, the model allows both wage offers and home-production values to change over time. Changing wage offers may account for a possible loss in human capital and/or changes in hours worked 2 Changing values of home production allow for capturing the idea that staying home with the baby is very important initially but less important later on. In this basic setting, we introduce the two interesting policy parameters: a maximum duration of job protection during

2. Our data do not allow us to disentangle the two channels as we only observed daily but not hourly wages. 
which women can return to their pre-birth job at the same wage; and a maximum duration of a fixed amount of cash benefits.

The model builds on Frijters and Van der Klaauw 2006) who structurally estimate a job search model with an outside option. Our structural model goes beyond Frijters and Van der Klaauw 2006) (i) because in our case the outside option (returning to the same employer) is timedelimited (while it is unlimited in their model); and (ii) because the value of home production changes over time.

We use this model to assess to what extent the behavioural framework can predict the impact of the three Austrian PL reforms on mothers' return-to-work behaviour. Conducting out-ofsample predictions on the basis of our structurally estimated parameters, we find that our model predicts return-to-work and return to same employer behaviour remarkably well. We also use the estimated parameters to estimate return-to-work behaviour under counterfactual policies: a regime with neither job protection nor benefits, a policy with only benefits, and a policy with only job protection. Our simulation results show that a system that combines both policy instruments generates more time for care immediately after birth and more employment in the medium run than systems that use just one or none of the two policy instruments. Thus, cash and protection complement each other in ensuring time for care and insuring pre-birth labour market investments.

Most of the previous literature has found that more generous PL provisions tend to delay women's return-to-work. However, evidence of the relationship between duration of leave and women's labour market outcomes is mixed. A key empirical challenge has been to find exogenous variation in leave-taking by mothers. Many studies use variation in leave availability across employers or leave-taking by employees. However, most of these studies suffer from several sorts of biases due to unobserved differences between mothers who had access to maternity leave and mothers who do not and between mothers taking longer and shorter leaves. The use of more plausibly exogenous variation in the length of parental leave has been limited.

Studies that focus on the U.S. have examined the impact of the 1993 U.S. Family and Medical Leave Act (FMLA), which guarantees a job protected unpaid maternity leave of 12 weeks to women working for companies with 50 or more employees. These studies find only modest or no effects of mandated protected leave on the length of parental leave and subsequent employment, although they do find some positive impacts on job continuity (see, e.g., Klerman and Leibowitz. 1997; Klerman and Leibowitz, 1999; Waldfogel, 1999; Baum. 2003). In addition, most of these studies found no significant effects on wages (see, Waldfogel, 1999; Baum. 2003; Hashimoto et al. 2004). Nevertheless, these results are difficult to generalize to other contexts given the relatively short length of job protected leave guaranteed by FMLA and the fact that, in most cases, this policy does not have a significant impact on duration of maternity leave taken by mothers. Moreover, the population affected by FMLA accounts for less than 50\% of the private sector workers in the U.S. (see Waldfogel, 1999).

PL rules in Canada and Europe are more generous and hence more likely to have an impact on women's labour supply and career prospects. Baker and Milligan 2005) exploit variation in PL provisions over time and across Canadian provinces and find that both short and long mandates increase job continuity. However, only long leaves appear to increase the amount of time that mothers spend away from work. Ruhm 1998) compares employment rates and wages of men and women using panel data of European countries, and finds that longer leave mandates are associated with higher female employment but lower relative wages. Ejrnaes and Kunze 2006 investigate the role of PL on the family wage gap exploiting exogenous variation in the length of PL generated by policy changes in the German system. They find that longer PL duration leads to detrimental effects on employment and wages for women. In contrast, Schönberg and Ludsteck 2007) study the same German reforms and find only minor effects on employment rates and mixed effects on wages. Lalive and Zweimüllen 2009) study the effects of the 1990 and 1996 
Austrian reforms and find that extensions of PL increase fertility. They also examine the impacts on earnings and employment but neither do they analyse the separate effects of benefits and job protection nor do they examine additional labour market outcomes, such as experience, tenure, and unemployment.

This article goes beyond the existing literature in two main dimensions. First, we examine the relative importance of duration of job protection and cash benefits by studying alternative PL policy mixes exploiting a series of major policy changes in Austria since the early 1990s. Our empirical analysis sheds light on mother's behaviour in the short run, and it also generates evidence on their medium run labour market outcomes through a comprehensive analysis of mother's outcomes over time since the child's birth. Second, on the basis of a structurally estimated a non-stationary job search model, we are able to study in detail the impact of cash benefits and job protection both in isolation and in interaction with each other. The model also turns out to be a powerful tool to undertake out-of-sample predictions and to examine the impacts of counterfactual PL regimes.

The remainder of the article is organized as follows. Section 2 discusses the institutional background and lays out the relevant details of the Austrian PL reforms. In Section 3, we introduce the data and present some descriptive characteristics of our samples. Section 4 discusses identification and presents reduced-form evidence on the impact of policy parameters on returnto-work decisions and medium-run labour market success. Section 5 presents the theoretical framework and a structurally estimated job search model. In Section 6, we use the model to make out-of-sample predictions and counterfactual experiments. Section 7 concludes.

\section{INSTITUTIONAL BACKGROUND AND PL REFORMS}

In this section, we briefly describe the institutional background of Austria concerning family policies in general and PL policies in particular. We then discuss the reforms to the Austrian PL systems of 1990, 1996, and 2000. We argue that these reforms provide a quasi-experimental situation allowing us to identify the causal effect of PL duration on labour market outcomes.

\subsection{Parental leave policies before July 1990}

Austrian family policy rules divide the time immediately before and after the birth of a new child into a period of maternity protection and a period of parental leave. Maternity protection starts 8 weeks before the estimated date of birth and lasts for 16 weeks ( 24 weeks for premature, multiple, and caesarian-section births). During this protection period, mothers get a government transfer that replaces $100 \%$ of the pre-birth wage (i.e. average labour earnings during the last 3 months prior to benefit take-up). The maternity protection rule intends to protect the health of both mother and child by giving mothers the maximum incentive to stay off work around childbirth.

The period of maternity protection is followed by the $P L$ period during which the mother (i) gets a flat government transfer and (ii) enjoys job protection. The government cash benefit amounts to roughly $35-40 \%$ of female net median income, is independent of household income and not taxed 3 Cash benefits are conditional on staying at home with the child and are terminated when the mother returns to work before exhausting the maximum PL duration. Job protection means the mother has the right to return to the same job at her previous employer and cannot be 
fired during the first 6 weeks after returning from parental leave. Thereafter, the regular advance notice rules apply 4

According to the rules that were in place before July 1990, eligibility to PL benefits was tied to employees who had contributed a minimum number of months to the social security system. This work requirement amounted to at least 52 weeks within the 2 years prior for a first birth; and to 20 weeks within the last year for second- and high-order births and for mothers younger than the age of 25 years. Self-employed mothers and mothers working in own-family firms and farms were not eligible.

\subsection{The parental leave reforms of 1990, 1996, and 2000}

While maternity protection rules remained roughly unchanged, the parental leave system underwent major changes since the early 1990s. The first major reform was enacted on July 1, 1990. Before July 1990, the maximum PL duration ended at the day when the child turned 1 year of age. The 1990 reform extended both the maximum duration of job protection and the maximum duration of cash benefits by 1 year so that maximum PL duration ended 24 months after birth. The reform also introduced the possibility to share the second year of the parental leave by both parents and/or spend a part-time leave (i.e. reducing work-time by $50 \%$ and drawing only $50 \%$ benefits; either both parents during the second year, or one parent during the second and third year). However, while it turned out that mothers reacted strongly to increased leave durations neither take-up of parental leave by fathers nor take up of part-time leave was substantial. The 1990 reform was mainly intended to help young mothers in combining childbearing and working. It was enacted in times when the economy was in a boom and the government did not face any severe budget constraint. Since take-up was unexpectedly high, this reform turned out to be quite costly.

The second major reform was enacted on July 1, 1996. It left the maximum duration of job protection unchanged. While the maximum duration of cash benefits remained unchanged as well, a sharing rule among both parents was introduced so that cash benefits could only be drawn for the maximum duration if each parent took a leave of at least 6 months. In practice, PL takeup by fathers was very low. Hence, the introduction of the sharing rule effectively reduced the maximum benefit duration from the day when the child completed 2 years of age to the day when the child completed 1.5 years of age. The 1996 reform also implemented some minor changes to previous work requirement rules. The work requirement, within the last year prior to the birth, was reduced from 20 to 16 weeks for mothers under the age of 25 years; and was increased from 20 to 26 weeks for second and higher-parity births. A major intention behind the 1996 reform was budget cuts. Since the 1990 reform turned out to be quite costly (and since Austria wanted to join the EU and had to obey the EU deficit/debt rules), the government was under severe pressure. The 1996 PL reform was part of a series of changes to welfare programmes in an effort of the government to bring down the budget deficit and public debt5

The third major reform took place when Austria moved from a parental leave system to a child benefit system. The key innovation was that eligibility to cash benefits was no longer conditional on pre-birth labour market history. All mothers (including the self-employed and mothers out of the labour force) became eligible for cash benefits. Like the 1996 reform, the 2000 reform left

4. The job protection rule of the Austrian PL system generates substantial firing costs. Since the advance notice period is at least 3 months, an employer who does not want to re-employ the mother after her baby-break has to pay her pre-birth waged for at least 4.5 months.

5. It is important to note that none of the other reforms to the welfare system was tied to the child's date of birth so that they are expected to affect all women equally. 
the maximum duration of job protection unchanged but introduced several major changes to the cash benefit part of the system. It increased the maximum duration of cash benefits until the day before the child completed its third year of age keeping the parental sharing rule. Hence, the maximum duration of cash benefits effectively increased from 18 months to 30 months. Finally, the reform also allowed mothers to draw cash benefits and work-as long as yearly earnings did not exceed 14,600 Euros per year. This policy change was made public on August 7, 2001, and became effective for children born on or after January 1st 2002. In order to ensure equal treatment of parents, parliament also allowed parents who were on parental leave on August 7, 2001 and gave birth after July 1st, 2000 to extend PL payments to 30 months (36 months if shared) provided that their annual income was below 14,600 EUR. While the increase in the earnings threshold for benefits eligibility allowed some post-July mothers to work while receiving benefits after month 18, it is probably of second-order importance in analysing medium-run labour market outcomes.

The three reforms were unlikely to be anticipated at the time of conception for parents who gave birth within a few months around the policy change. For example, the 1990 reform act was first mentioned in November 1989, i.e. 7.5 months before it was implemented. Moreover, this reform only passed the Austrian parliament in April 1990 and in January 1990 it was still unclear whether the reform would be implemented at all (and, if so, when). The 1996 reform followed a similar political history with high uncertainty regarding its details and likelihood of implementation around the last months before it took effect. Hence, self-selection into the preand post-reform regimes through fertility decisions is highly improbable. Still, parents could selfselect into the more generous PL regimes by rescheduling planned caesarian sections or speeding induced labour. We address these issues in the next section where we discuss the empirical strategy.

The situation of the 2000 reform is different, as this reform was implemented retrospectively on January 1, 2002 but the new rules applied for all mothers whose birth took place on July 1, 2000 or later. Therefore, manipulation of conception or delivery dates can be ruled out completely. However, a comparison of mothers who gave birth immediately before and immediately after July 1, 2000 might be affected by other factors. The reason is that mothers who delivered in July/Augst 2000 may have made their labour supply choices on the basis of pre-July 2000 PL rules (and may have committed themselves towards their employers). A second potential confounder for the 2000 reform is that results might not only be the result of extended PL durations but can also be generated by the introduction of generous earnings limits, that allowed mothers under the new regime to draw benefits while working. Both possible confounders induce mothers to work more under the post-July 2000 rules. While we cannot do much about this, we show in Section 4.2 that the group returning to work due to these two factors appears to be rather small. In any case, we note that results obtained from contrasts based on the 2000 reform are most likely to be downward biased (i.e., they provide a lower bound for the PL treatment effect).

\subsection{Other fertility-related family policies}

Besides PL benefits, fertility-related family policies in Austria consist of a broad set of measures that we only briefly discuss here. A further transfer to which parents are eligible are child allowances (Familienbeihilfe). There is universal eligibility to these benefits (meaning that all parents with sufficiently long residence in Austria are eligible) and parents are eligible as long as kids are still in the education system. Benefit levels depend on the age of the child. The tax system has deductions for children (Kinderabsetzbeträge) that increase with the number of children. Furthermore, before 1997, parents were eligible to a birth benefit (Geburtenbeihilfe) of 
1090 Euros, paid out to mothers in several steps upon medical inspections between the child's birth and its fourth birthday. The supply of child care facilities for small children was rather low in the 1990s and early 2000s. According to the OECD (Employment Outlook 2001), the proportion of children under the age of 3 years enrolled in child-care arrangements was only about $4 \%$ in 1998 which is very low by international standards 6 In contrast, informal care arrangements or extended family care arrangements are very important for dual earner families in Austria with children under the age of 3 years.

While the most significant changes in fertility-related family policies during the 1990s concerned changes in PL legislation, several other minor changes were made with respect to other family policies. In 1997 the birth benefit was abolished. In 1998, there was a major effort by the central government to improve the supply of childcare facilities in public kindergardens (Kindergartenmilliarde). While this was a major effort of the government, it was targeted towards the 3-6 years old children rather than children below the age of 3 years so that this policy did not directly interfere with changes in PL rules. Moreover, it is important to note that eligibility to none of the additional family policies changed discontinuously on July 1st (the date of PL regime changes). Therefore, they are not expected to confound our estimates for the effects of the alternative PL regimes 7

\section{DATA}

We use data from the ASSD. The ASSD consists of administrative individual register data collecting information relevant for old-age social security benefits. As these benefits depend on individuals' earnings and employment histories, the data set reports individuals' complete employment histories since 1972 for the universe of Austrian private sector workers. Furthermore, not only employment histories, but also time with childbearing and rearing ('Kinderersatzzeiten') are relevant for old-age social security benefits. This is why the ASSD also reports highquality information on the number of births by female employees with previous social security contributions.

The ASSD has several advantages which will be of particular importance for the empirical strategy developed below. First, the data set covers the universe of the private sector employees (about $80 \%$ of the total workforce) in Austria, implying that we can rely on large samples, even when very specific groups are considered. Second, the data reports, on a daily basis, the occurrence of a birth and take-up (and durations) of maternity protection and parental leave since the year 1972. This allows us to determine precisely both the PL eligibility status as well as the maximum duration of PL of mothers. Third, as all employment and earnings over an individual's life cycle are reported in the data, we can look in a very detailed way at the joint distribution of labour supply behaviour and earnings of mothers over extended time periods.

6. For Denmark, Norway, and Sweden $64 \%, 40 \%$, and $48 \%$, respectively. Germany and southern European countries have similarly low levels of child care facilities for kids under the age of 3 years. These figures include both public and private child care provision such as group care in child-care centres, residential care, childminders based in their own home, care provided by person who are not a family member; see OECD Employment Outlook 2001.

7. We also examined how mother's return to work behaviour is affected by other policies to assess whether the PL reforms might have a different effect according to additional policies faced by mothers. For this purpose, we examined whether the abolition of the birth benefit mentioned above affected mother's time on leave (see Brunner and Kuhn 2011 for more details on this reform). Our results show no significant difference in return-to-work profiles for mothers who gave birth before and after the abolition of the birth benefit. This shows that mother's time on leave was not affected by the birth benefit. 
To examine the impacts of the PL reforms on return to work behaviour and labour market outcomes, we select mothers who gave birth 2 months before and 2 months after each policy change. We select all women who are potentially eligible for PL entitlements using the same criteria for all years. Since we are interested in post-birth labour market outcomes of women after a PL, we apply a stricter criteria than the PL eligibility required by law, and restrict the sample to women employed in the year prior to giving birth. For each reform, we define a treatment and a comparison group (births in May and June versus births in July and August of the respective year when PL rules changed). We further stratify the sample by parity (number of times a woman has given birth) and perform a separate analysis for women giving birth for the first time and for women giving birth at higher parities. 2005 is the last year available to us with earning records. We therefore limit the analysis on medium-run labour market performance to the fifth year after the child's birth to provide a common time period to analyse and compare the effects of the three reforms. Because the ASSD covers the universe of all individuals who, at some previous date, paid social security contributions, we end up with a sufficiently large data set: 10,815 mothers who gave birth at parity one (i.e. the first birth in a woman's life) between May and August 1990; 10,514 mothers who gave birth at parity one between May and August 1996; and 9103 mothers who gave birth at parity one between May and August 2000. The sample sizes for mothers giving birth at higher parities are 4449 mothers in 1990; 3856 mothers in 1996; and 4351 mothers in 2000. We also add cohorts who gave birth during the same months in the year preceding each reform. This raises our final sample size for parity one to 21,507, 21,146, and 18,345 mothers in 1990, 1996, and 2000, respectively. Our final samples for parity higher than one include 8575, 7754, and, 8541 mothers.

\section{REDUCED FORM EVIDENCE}

This section first discusses how we identify reduced form effects of the three policy changes. The section then estimates the effects of the reforms on return to work and medium-run labour market outcomes.

\subsection{Econometric method}

Using the samples described above, we investigate how strongly duration of PL changes as a function of date of birth. Panels (a)-(c) of Figure 1 report average durations of benefit receipt within the first 2 years after child's birth for mothers giving birth between May 1 and August 30 in 1990 and in 1996 and for the first 30 months after child's birth for mothers giving birth between May 1 and August 30 in 2000. The figures show very clearly that benefit take-up is highly responsive to changes in PL regulations 8 For example, mothers who gave birth before July 1990 received PL benefits on average for 10 months. In contrast, the corresponding number for mothers who gave birth after June 1990 is, on average, about 20 months. Importantly for our empirical strategy, there is no trend in average PL durations within the period before the PL change and within the period after the PL change for none of the three years of policy changes.

Panels (d)-(f) of Figure 1 plot benefit take-up for the cohort of mothers giving birth between May 1st and August 30th of the year preceding each of the reforms. As clearly seen, there is no discontinuity in the length of parental leave around July 1st in years when there was no policy change. This suggests that exposure to the new PL regimes is the source of the discontinuous

8. While our data set does not report the PL eligibility status directly, we observe actual PL take-up. Note that PL take-up is itself an endogenous variable. However, as most mothers use up the eligibility period, this indicator is informative on the treatment intensity. 

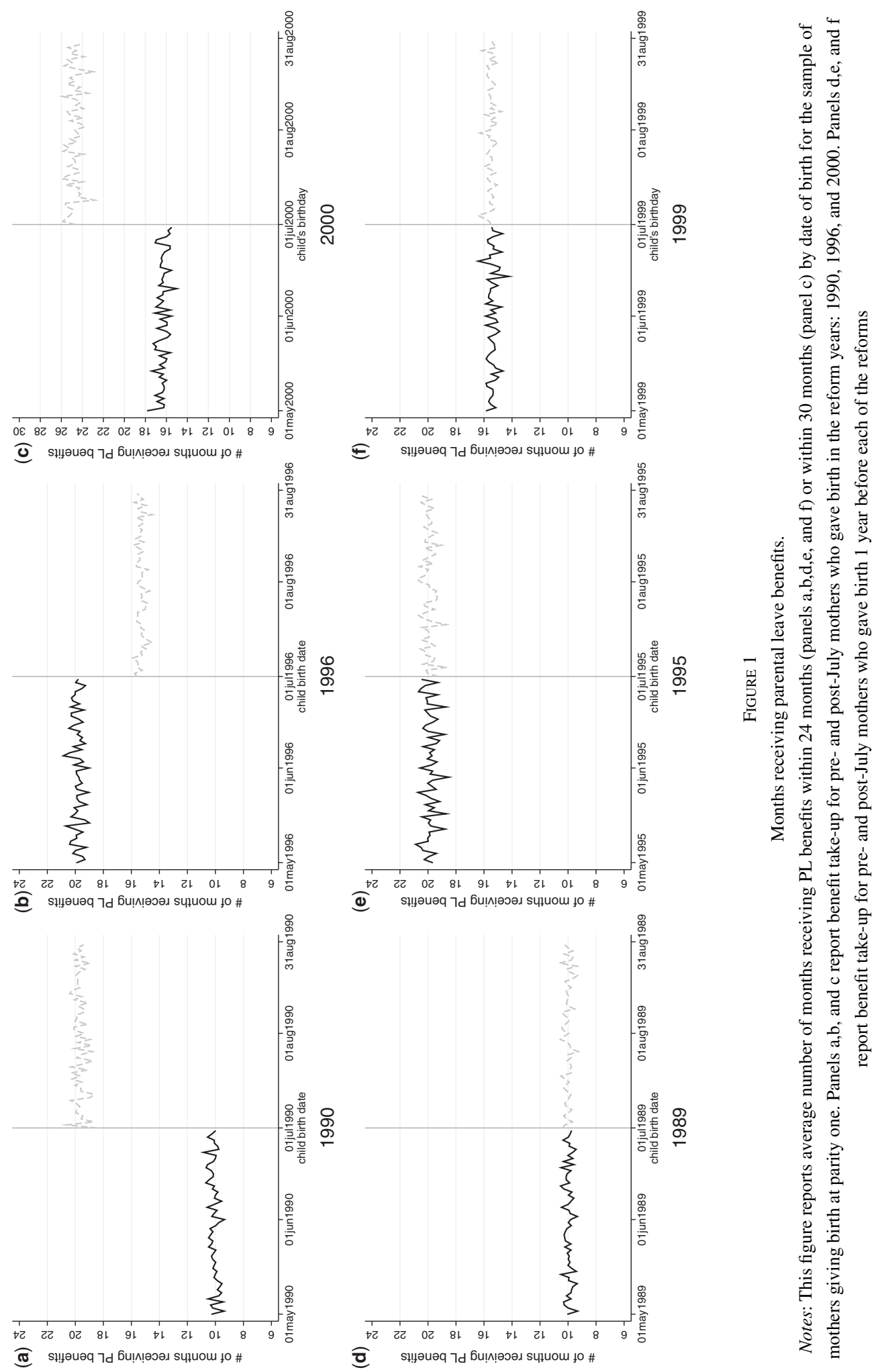
break between June 30th and July 1st and not any type of seasonality in childbearing or labour market behaviour of mothers.

We use a regression discontinuity design to assess the average causal effects of duration of PL benefits and job protection on mothers' return-to-work decisions and subsequent labour market performance. Let $T$ denote the date of birth of a child, $Y$ the labour market outcome of interest (e.g., time to return-to-work, employment status, earnings, etc.) and $D$ a treatment indicator, with $D=1$ for mothers giving birth under the more generous policy regime in the relevant year (post-July 1st in 1990 and 2000 and pre-July 1st in 1996) and $D=0$ otherwise. Assignment to treatment is a discontinuous function of the date of birth $T$. That is, $D=I\left(T>=t_{0}\right)$ for the 1990 and 2000 sample and $D=I\left(T<t_{0}\right)$ for the 1996 sample, where $t_{0}$ is the day of policy change (July 1st of the relevant year).

Evidence presented above shows that assignment to treatment changed discontinuously between June 30 and July 1 . Thus $E\left(D \mid T=t_{0}+\epsilon\right)=1$ and $E\left(D \mid T=t_{0}-\epsilon\right)=0$, i.e. assignment to treatment is "sharp" in the terminology of Hahn et al. 2001) 9 An intuitively appealing contrast that infers the causal effect of extended PL benefits is the following:

$$
E\left(Y \mid t_{0} \leq T \leq t_{0}+\epsilon\right)-E\left(Y \mid t_{0}-\epsilon \leq T<t_{0}\right)
$$

It can be shown that for $\epsilon>0$ sufficiently small, this contrast identifies the average effect of offering extended PL benefits on the outcome of interest Hahn et al. 2001).

In the empirical analysis, we report results based on $\epsilon=61$ calendar days. More precisely, we compare between mothers who gave birth in July/August and mothers who gave birth in May/June. To control for any differences in demographic characteristics or labour market performance between mothers who give birth before or after July 1st, we also add a pre-reform cohort of mothers who gave birth during the May-August interval. The causal effect of the extension of PL benefits is therefore attained by the difference between the outcomes of mothers who gave birth in July/August versus May/June in the year of the policy change (i.e., 1990, 1996, and 2000) relative to the difference in outcomes of mothers who gave birth in July/August versus May/June in the year preceding the policy change (i.e., 1989, 1995, and 1999). Namely, we identify the causal effects of PL extensions using a difference-in-differences regression discontinuity (DID-RD) approach. We estimate the following model for each reform separately allowing all coefficients to differ in each reform.

$$
y_{\text {imt }}=\beta_{0}+\beta_{1} D_{m} * \text { reform }_{t}+\beta_{2} D_{m}+\beta_{3} \text { reform }_{t}+x_{i}^{\prime} \gamma+z_{\text {imt }}{ }^{\prime} \delta+\epsilon_{\text {imt }}
$$

where $y_{\text {imt }}$ is the outcome of mother $i$ who gave birth in month $m$ of year $t ; D_{m}=1$ for the months of the more generous leave regime; reform $_{t}$ equals one for the reform years $(1990,1996$, or 2000); $x_{i}$ is a vector of mother's characteristics that includes mother's age at birth and the following indicators of mothers' labour market performance measured 12 months before child's birth: tenure, experience, months of unemployment, cumulative income (overall income earned up to 12 months before the child's birth), and daily wage, and indicators for industry, region, and white collar. We also adjust for changes in macroeconomic conditions at time of re-entry into the labour market by controlling for the local unemployment rate in the region of pre-birth employment. However, since time of re-entry is a choice variable, we focus on differences in conditions at time of re-entry that are driven by exogenous factors. Namely, we control for the unemployment rate at the end of benefit and job protection periods. These covariates are included

9. Note that in the analysis, we treat time as discrete with the smallest time unit equal to 1 day. This guarantees, that the density of births at $t_{0}$ is non-zero. 
in vector $z_{\text {imt }} 10$ Doing so ensures that effects on labour market outcomes are not driven by changes in the business cycle at time of re-entry. In practice, our results (not reported here but available upon request) are not sensitive to the adjustment of macroeconomic conditions at the time of re-entry, suggesting that treatment and comparison mothers faced a similar economic environment.

There are several reasons why a comparison between mothers giving birth in May/June and mothers giving birth in July/August is informative on the causal effect of duration of PL benefits. First, observed characteristics of the two groups are very similar. This is what we would expect if assignment to treatment is almost as good as randomly assigned. Table 1 shows that the two groups are quite comparable in terms of their pre-birth background characteristics and pre-birth labour market outcomes for the three policy years with the exception of a few characteristics such as age in 1990, for instance. Differences get smaller, however, once we condition on age and include the pre-reform cohort to control for any seasonal differences between mothers who give birth between May/June and July/August. Importantly, pre-birth job characteristics, like average earnings per day and white-collar employment, are almost identical between the two groups 11 As shown in Appendix Table A.1 we also find no differences between pre- and post-July mothers who gave birth at parities greater than one. Nevertheless, while pre- and post-July mothers are similar, they are not completely identical. Our analysis below will therefore control for these individual pre-birth characteristics.

A second feature that justifies our approach is that not only treated and comparison mothers are similar in terms of pre-birth characteristics, but they also face virtually identical macroeconomic and labour market conditions before and after giving birth. On average, July to August mothers gave birth to the child that defines their treatment status only 2 months after May to June mothers. Moreover, by including a cohort of mothers who gave birth in the pre-reform year, we further assure that any seasonal differences in labour market conditions or labour supply costs (e.g., holidays, vacations, seasonal work, childcare enrollment, etc.) correlated with month of birth will be differenced out 12

A third reason that justifies the validity of our identification strategy refers to the way the treatment status is assigned to individuals. As we focus on births that took place during a relatively short period (from May until August), this comes close to a process of random assignment to treatment. As described in the previous section, anticipation of the reforms was minimal. However, even if anticipations of the reforms by the time of conception is very unlikely, some parents could

10. For May to June 1990 mothers and for May to June 1989 mothers, we control for the unemployment rate in pre-birth region of employment 12 months after the child's date of birth. For July to August 1990, May to August 1995, and May to June 1996 mothers we control for the local unemployment rate 24 months post-birth. For July to August 1996, May to August 1999, and May to June 2000 mothers we control for the local unemployment rate 18 and 24 months after giving birth, and for July to August 2000 mothers we condition on the local labour market situation 24 and 30 months after giving birth. Estimates are not sensitive to including these controls.

11. There are some differences in pre-birth labour market outcomes in 1990. However, these differences are small relative to the outcome means and are of inconsistent signs across outcomes. For example, post-July mothers in 1990 seem to have pre-birth daily earnings that are about $1 \%$ higher relative to pre-July mothers. On the other hand, they are less likely to work in white-collar occupations. In 1996 and 2000, we see no differences in pre-wage earnings.

12. We find some seasonality as we see that in all years (including those with no reform), pre-July mothers returnto-work a bit earlier. As a result, estimates based on samples that include only mothers who give birth in the reform year (not reported in the article but available upon request) show that the impacts of the 1990 and 2000 reforms on time until return-to-work are slightly larger while the impacts of the 1996 reform are slightly smaller than the results obtained when using also the pre-reform year. Despite that, estimates for outcomes measured in year 5 after birth, are not sensitive to the exclusion of mothers from the pre-reform year. We therefore prefer to correct for seasonality in our analysis by contrasting differences between pre- and post-July mothers who gave birth in the reform year with differences between equivalent cohorts who gave birth in the pre-reform year. 


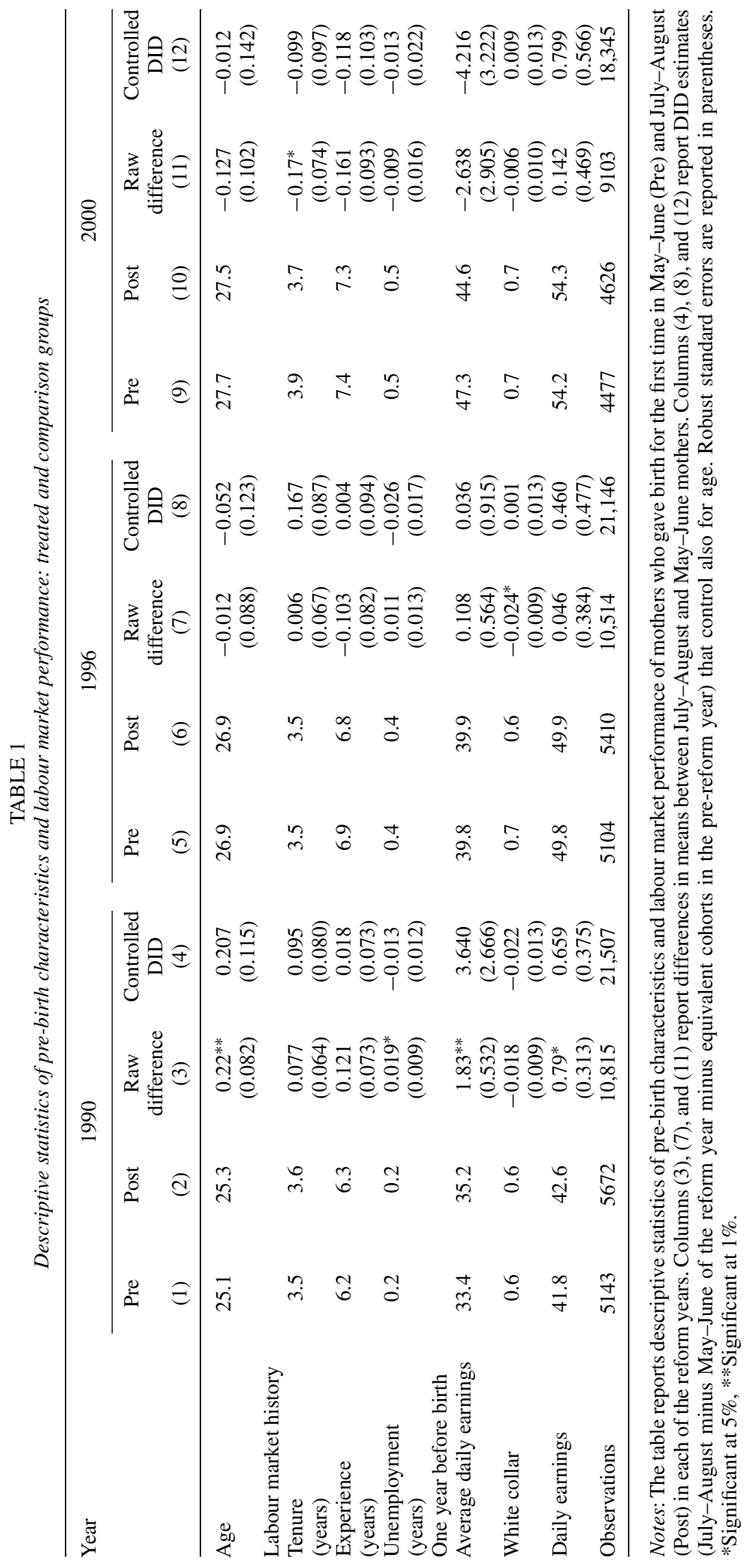


still self-select into the more generous PL regimes by rescheduling planned caesarean sections or induced labour. We assess the possibility of such manipulation as follows 13 First, we analyse the frequency of births by date during the months of May-August for the years of the policy changes and do not find any evidence of a spike in births on the days surrounding July 1st. Moreover, we find that the distribution of births by date of birth in years of policy changes highly resembles the distribution observed in years where there was no policy change. Second, because manipulation of birth dates is more likely to exist around the reform date, we estimated alternative models where we allowed for differential trends in time to policy change. Specifically, we estimated an additional model from a sample that includes mothers who gave birth 3 months around the cutoff date while controlling for differential time trends for births before and after the cutoff date and for births in the reform and the pre-reform year. We also estimated models based on an alternative sample that includes all mothers who gave birth during the year of the policy change and allows for differential quadratic time trends. Estimates from these models are less precise than those reported here but they are highly similar to our main results. In addition, we also re-estimated all models while excluding mothers who gave birth during 1 or 2 weeks around the cutoff date. Estimates from these samples are virtually identical to those obtained when using the full sample and reported below. As an additional test for the robustness of our results, we also defined some placebo treatments by assigning a treatment status to cohorts of mothers who gave birth in nonreform years. Estimates from these regressions showed no significant impacts for these placebo treatments. Finally, we estimated alternative models restricting the sample to different cutoffs around the policy change: 1.5 months, 3 months. All estimates are highly similar to our main results.

\subsection{Return-to-work decisions}

In this section, we analyse the effects of changes in duration of the benefit and job protection periods on return-to-work decisions. We begin by reporting results based on mothers of first-born children (parity one). The advantage of focusing on parity one is that PL eligibility is almost universal as most of these mothers were working prior to giving birth. In addition, their pre-birth labour market history is more informative about their skills and earnings capacity. On the other hand, it is important to note that since about half of these women give birth to at least one more child during the period of interest, our results are also influenced by fertility interactions. To asses the relative role of fertility interactions, we also examine the impacts of the PL reforms among women who give birth at higher parities. Results for mothers at higher parities are qualitative similar to those reported here and are reported in Appendix Tables A.2 and A.3

Figure2 2 plots Kaplan-Meier failure functions for time until return-to-work for mothers giving birth before July 1990. The vertical line at month 12 denotes the end of the benefit and job protection period. Roughly $10 \%$ of the pre-reform mothers return-to-work within 3 months after birth. Thereafter, the proportion returning to work increases gradually reaching a level of $18 \%$ before the child's first birthday. This implies that more than $80 \%$ of mothers of newborn children fully exhaust their PL entitlements. At the child's first birthday, the proportion of mothers back at work increases sharply to $43 \%$. Thereafter, the proportion back at work increases steadily reaching a level of almost $80 \%$ after 5 years.

How does the extension of job protection and cash benefits affect mothers' return-to-work behaviour? The 1990 reform, which guaranteed job protection for 24 months, had the potential of increasing the fraction of mothers returning to work within the job protected period. On the

13. Results from these additional tests are not reported here but are available upon request. 


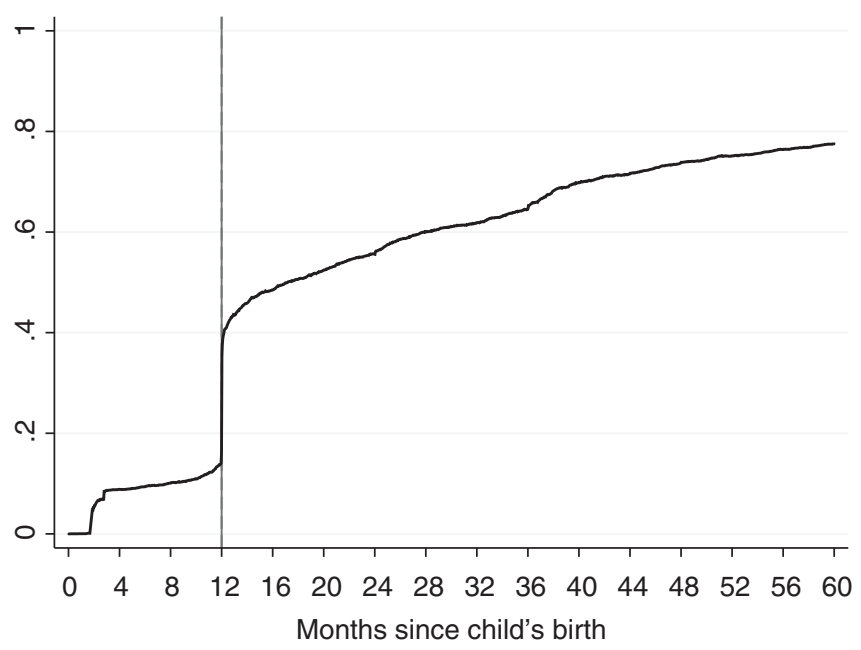

FIGURE 2

Return-to-work before July 1st 1990.

Notes: This figure shows the proportion who have returned to work at or before $t$ months after child's birth. The sample includes mothers giving birth at parity one between May 1st and June 30th 1990. Women giving birth before July 1990 are eligible for 12 months of job protection and 12 months of benefit payments

other hand, since benefits were also extended by the same amount of time, return-to-work dates are likely to be delayed. Figure 3 plots return-to-work profiles for mothers giving birth before and after the policy change 14 The solid line plots profiles of pre-reform mothers and the dotted line plots profiles of post-reform mothers. The vertical lines denote the end of the job protection and benefit period of the two regimes.

As expected, return-to-work behaviour of mothers who stay on leave for less than 12 months is almost unchanged by the PL reform. These mothers are strongly attached to the labour market and their return-to-work is not bounded by the PL policies. A sizable gap in the behaviour of pre- and post-July mothers appears at month 12 when the two groups of women face a different policy environment. While at the term of 12 months a significant share of pre-reform mothers return-to-work, a sizeable share of the post-reform women delay return-to-work and exhaust the 2 years of extended leave benefits. At the child's second birthday, when benefits and job protection end, a large fraction of post-reform mothers return-to-work. Interestingly, the 12-month extension of job protection and benefits leaves the proportion of mothers who return-to-work within the job protected period almost unaffected. Overall, the extension of PL entitlements shifts the return-to-work profile by about 12 months while preserving its original shape. Interestingly, the return-to-work profile is also shifted for mothers who return-to-work after PL benefits and job protection are exhausted. This shift implies that the share of women who return-to-work is still lower (by about 7 percentage points) for the post-reform group than for the pre-reform group even 60 months after birth. Nevertheless, as we will discuss in Section 4.3 , this delay in return-to-work does not translate into a reduction in earnings in the medium run.

14. Return-to-work profiles are adjusted for seasonality. We do this by subtracting from the profile of the comparison group in the reform year the difference in return to work profiles between the comparison and the treated group in the pre-reform year. 


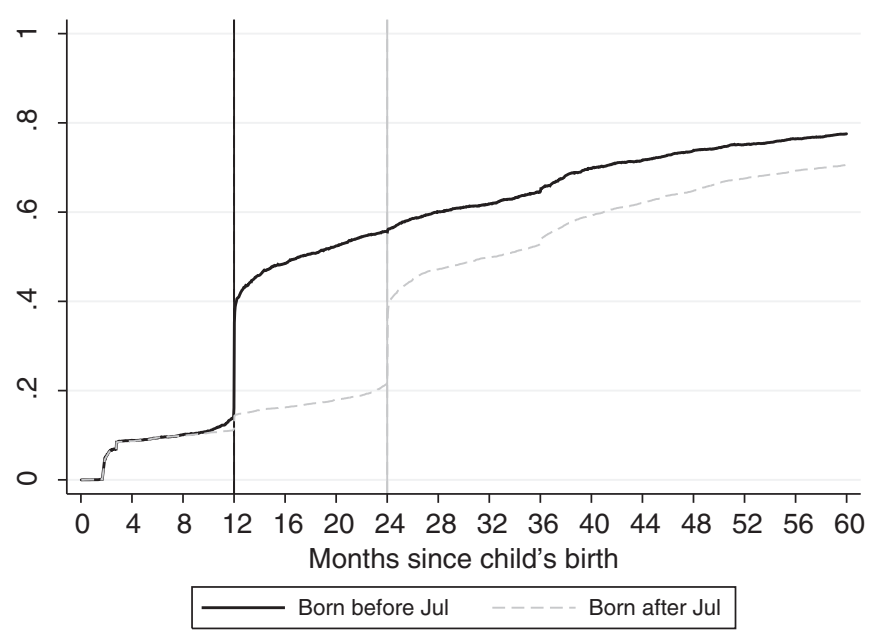

FIGURE 3

Return-to-work with extended benefits 1990.

Notes: This figure shows the proportion who have returned to work at or before $t$ months after child's birth. The sample includes mothers giving birth at parity one between May 1st and August 30th 1990. Women giving birth before July 1990 are eligible for 12 months of job protection and benefit payments. Women giving birth after July 1990 are eligible for 24 months of job protection and benefit payments. Return-to-work profiles are corrected for seasonality. See footnote 14 for details

We have seen in Figure 3 that mothers delay their return to work considerably as a response to an extension of PL benefits and the job protected period. A natural question is whether delays in return to work were induced by the extension of the job protected period, by the extension of benefits, or both. The 1996 and 2000 reforms allow us to shed light on this question as both policy changes affected the duration of PL benefits but not the duration of job protection. Figure 4 (panels $\mathrm{a}$ and $\mathrm{b}$ ) plots Kaplan-Meier failure functions for return-to-work profiles of mothers giving birth before and after the 1996 and 2000 reforms. The 1996 reform reduced the duration of benefits to 18 months. This reform allows discussing the role of paid job protected leave as opposed to unpaid job protected leave. The 2000 reform extended PL benefits by 12 months thus adding 6 months of paid protected leave and 6 months of paid unprotected leave.

Panel a of Figure 4 shows that the shorter benefits after the 1996 reform induced a large fraction of mothers to return-to-work earlier. The return-to-work profile is shifted backwards. However, compared to the expansion of the 1990 reform, the shift is less pronounced. This seems reasonable as the 1996 reform shortened the duration of benefit payments but left the duration of the job protected period unchanged. About $26 \%$ of the post-reform mothers return-to-work exactly at month 18 when benefits are exhausted. Still, there is a sizable group of mothers (12\%) who stay at home beyond the exhaustion of benefits but return within the period of unpaid job protected leave. About $4 \%$ of the mothers return-to-work exactly at the end of the job protected period. Return-to-work responses to the 1996 reform suggest that while benefits and job protection have independent effects in delaying women's return-to-work, the impact of benefit duration appears to be more significant. This conjecture is further supported by changes in return-to-work profiles induced by the 2000 reform.

Panel b of Figure 4 plots return-to-work profiles for pre- and post-reform mothers in 2000. Post-July mothers received 12 extra months of benefits but only 6 of them were job protected. Clearly, the post-reform cohort displays a return-to-work profile that is consistent with the changes 
(a)

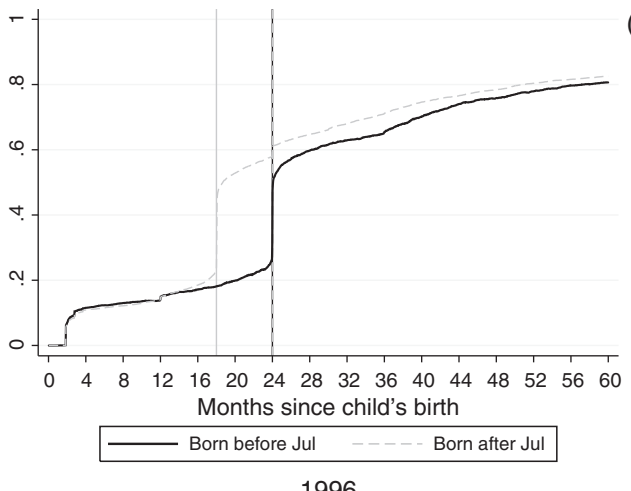

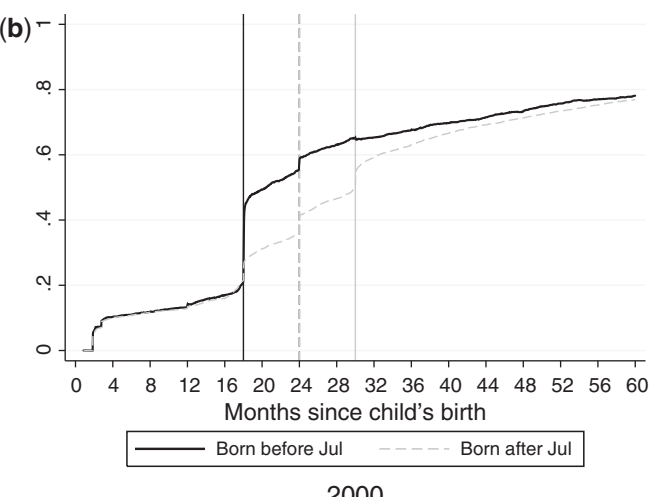

2000

FIGURE 4

Return-to-work profiles for the 1996 and 2000 reforms.

Notes: This figure shows the proportion who have returned to work at or before $t$ months after child's birth. The sample includes mothers giving birth at parity one between May 1st and August 30th of 1996 (a) and 2000 (b). Women giving birth before July 1996 are eligible for 24 months of job protected and paid leave. Women giving birth after July 1996 and before July 2000 are eligible for 24 months of job protected leave but only 18 months of paid leave. Women giving birth after July 2000 are eligible for 24 months of job protected leave and 30 months of paid leave. Return-to-work profiles are corrected for seasonality. See footnote 14 for details

imposed by the PL reform. In this case, the return-to-work profile is shifted forward responding to the extension of the benefit period. Again, we see in this case that mothers respond to both duration of benefits and job protection. We also observe a relatively small proportion of post-July mothers (about $8 \%$ ) who return-to-work exactly at month 18, the first month when the income ceiling was raised enabling mothers to work without losing the right to withdraw benefits. The share returning to work at month 24 , when job protection ends, is similar to the corresponding share in the pre-reform group. There is a further sizable group returning exactly when benefits are exhausted at month 30 , suggesting that duration of benefits even when not coupled with job protection induced some mothers to delay their return-to-work.

The above figures clearly show that mothers are highly responsive to both PL benefits and job protection, with benefits appearing to play a more important role. A larger proportion of mothers return-to-work when the benefit period ends before the job protection period than when job protection ends before benefits. We will see in Section5 that empirical return-to-work profiles match the predictions of our search model: reservation wages appear to be shifted upward to a larger degree by extension of benefits than extension of job protection 15

Table 2 summarizes the effects of PL extensions on return-to-work behaviour by reporting $D I D-R D$ estimates of the three reforms on total months at home (censored at 60 months), the likelihood of returning to work within 60 months, the likelihood of returning to pre-birth employer, and daily wages at first post-birth job. Each column reports estimates for a specific policy reform (i.e., 1990, 1996, and 2000). Outcome means for the cohort exposed to the less generous leave are reported in italics. In all cases, estimates contrast the cohort with the more generous leave (postJuly in 1990 and 2000 and pre-July in 1996) to the cohort with the more restricted leave (pre-July

15. We have explored whether responses to the parental leave reforms differ by marital status and couple's education (see Supplementary Appendix Section 1). The overall pattern of results is relatively similar to that reported for the overall group for most sub-groups. 
TABLE 2

The causal effects of the reforms on return-to-work

\begin{tabular}{lccc}
\hline Year of reform & 1990 & 1996 & 2000 \\
& $(1)$ & $(2)$ & $(3)$ \\
\hline Time at home (months) & $7.846^{* *}$ & $3.409^{* *}$ & $2.967^{* *}$ \\
(censored at 6 months) & $(0.539)$ & $(0.511)$ & $(0.547)$ \\
& 27.690 & 27.830 & 28.499 \\
Time until return to same employer (for those back to pre-birth job) & $7.263^{* *}$ & $3.517^{* *}$ & $3.688^{* *}$ \\
& $(0.435)$ & $(0.442)$ & $(0.457)$ \\
& 12.286 & 16.569 & 15.842 \\
Time until return to new employer (for those back to new job) & $6.873^{* *}$ & $2.804^{* *}$ & $2.794^{* *}$ \\
& $(0.645)$ & $(0.641)$ & $(0.722)$ \\
& 27.058 & 28.373 & 28.072 \\
Back within 60 months & $-0.071^{* *}$ & $-0.023^{*}$ & -0.009 \\
& $(0.011)$ & $(0.011)$ & 0.012 \\
& 0.786 & 0.826 & 0.795 \\
Back to pre-birth employer & $-0.038^{* *}$ & -0.003 & -0.021 \\
(censored at 6 months) & $(0.013)$ & $(0.014)$ & -0.014 \\
& 0.434 & 0.512 & 0.501 \\
Daily wage at 1st job after birth (in euro) & $1.566^{* *}$ & -0.771 & 0.546 \\
& $(0.540)$ & $(0.567)$ & $(0.956)$ \\
& 31.693 & 35.042 & 34.026 \\
Daily wage at 1st job after birth & 1.008 & -0.914 & 1.236 \\
(pre-birth employer) & $(0.764)$ & $(0.722)$ & $(0.947)$ \\
& 34.412 & 37.607 & 35.190 \\
Daily wage at 1st job after birth & $1.995^{* *}$ & -0.439 & -0.141 \\
(new employer) & $(0.712)$ & $(0.885)$ & $(1.761)$ \\
Number of observations & 28.339 & 30.857 & 32.034 \\
& 21,507 & 21,146 & 18,345 \\
\hline
\end{tabular}

Notes: This table reports DID-RD estimates for the impacts of the 1990, 1996, and 2000 reforms on mothers' returnto-work. The samples include all mothers who gave birth at parity one between May 1st and August 30th. Regressions compare differences in outcomes between the cohort exposed to the more generous regime and the cohort exposed to the less generous regime relative to the outcomes of pre- and post-July mothers who gave birth in the year preceding the reform. Estimates come from regressions that control for age at birth, and the following indicators for mothers' labour market performance measured 12 months before the child's birth: tenure, experience, months of unemployment, cumulative income, daily wages, and indicators for industry, region and white-collar occupation. Regressions also control for the unemployment rates in the region of pre-birth employment at the end of the job protection and benefit periods. Robust standard errors are reported in parentheses. Means of the comparison group (i.e., the group with access to the less generous regime) are reported in italics. *Significant at $5 \%, * *$ Significant at $1 \%$.

in 1990 and 2000 and post-July in 1996). The table reports estimates for mothers giving birth at parity one; estimates for mothers giving birth at higher parities are reported in Appendix Table A.2 estimates that control for differential time trends are reported in Supplementary Appendix Table 1.

As seen in the first row of the table, the 1990 extension of PL entitlements by 12 months extends time at home by 7.8 months. In 1996, 6 months of extra benefits extend time at home by 3.4 months. The 2000 reform, which added 6 months of protected benefits and 6 months of unprotected benefits, extended time at home by 3 months 16

Extending the period over which a person can remain on parental leave can have two conceptually different impacts. The first is a behavioural impact in the sense that parents change

16. The 2000 reform had a smaller impact than the 1996 reform for two reasons. First, the 2000 reform came into force only in summer 2001, i.e. after mothers had decided on the duration of parental leave. While all mothers were free to update and change their leave duration, we suspect that a substantial fraction of mothers were unwilling to change the initial arrangement. Second, the 2000 reform introduced a possibility to work while being on parental leave. As long as the total income from employment was below a threshold, women were allowed to get PL benefits and the additional earnings from work. Both factors explain why the extension of benefit duration of the 2000 reform had a smaller impact on time at home. 
their behaviour by re-optimizing under the new constraints. This is conceptually different from the case where the policy has no impact on the recipient's desired outcome but the policy change allows the parent to come closer to their desired outcome 17 This mechanical effect operates on mothers who want to return to the same employer. They are constrained to do so within the period protected by parental leave regulations. Extensions of parental leave duration loosen that constraint and will mechanically increase time at home. We report effects on time until return-towork for women who return to the pre-birth jobs versus women who take a new job to assess the role of this mechanical effect. Returns to the same employer will largely reflect the mechanical response, whereas returns to a new employer will not 18 Results show that PL extensions delay return-to-work to both pre-birth jobs as well as to new jobs, with a slightly larger impact on delays in returns to pre-birth jobs (see rows two and three of Table 2). The mechanical effect is indeed important. Yet, the results on returns to new employers suggest that un-constrained mothers are also adapting job search behaviour to the reforms.

We also see that the chances that mothers ever return to work within 5 years are somewhat lower in more generous regimes. We discuss this issue in more detail in the next section. An additional interesting finding is that extension of benefits and job protection generated only small changes to the relative chances that mothers return to their pre-birth employer or switch to new jobs after giving birth. We will turn back to this last finding in Section 6.1 where we outline a search model to examine the role of benefits and job protection and the interactions between these two policy instruments.

The last three rows of the table report the effects of the three reforms on daily wage at the first job after birth, daily wage at first job for those returning to their pre-birth employer, and daily wage at first job for mothers who started new jobs after birth. These estimates have to be taken with caution as they are affected by selection into employment and selection into pre-birth versus new jobs. In addition, we do not observe hours of work. Nevertheless, it is interesting to see that post-birth wages observed upon re-entry into the labour market are not affected by isolated extensions of benefits or job protection.

\subsection{Medium-run effects}

The purpose of this section is to discuss the medium-run effects of PL extensions on mothers' labour market performance after childbirth. We begin by presenting in Figure $5 D I D-R D$ estimates along with confidence intervals for the effects of PL extensions on cumulative outcomes such as labour market experience, months unemployed, and total earnings. The figure shows the dynamic effects of the reforms on these cumulative outcomes starting from the child's birth until year 5 after birth. The vertical lines denote the end of the job protection or the benefit period in the preand post-July regime. We also report in Table 3 the impacts on cumulative outcomes observed in year 5.

The first row of Figure 5 plots the impacts of PL extensions on labour market experience accumulated since the child's birth. It is clear that mothers in the more generous leave regimes accumulate fewer months of employment relative to mothers who gave birth in the less generous regime. However, it is interesting to see that the loss of labour market experience occurs entirely

17. We are grateful to a referee who pointed out this important distinction.

18. Note that it is not possible to draw causal conclusions from this stratification as the reforms are likely to affect the composition of the groups who return to pre-birth versus new jobs. We are nonetheless confident that this decomposition speaks about the likely magnitude of the mechanical effect since the number of women returning to the same employer does not change much (see below). Moreover, mothers who return to the same employer may also re-optimize given the new constraints. Their choices also reflect a behavioural effect on top of the mechanical effect. 
(a)

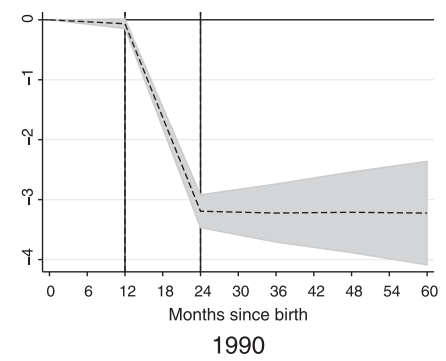

(d)

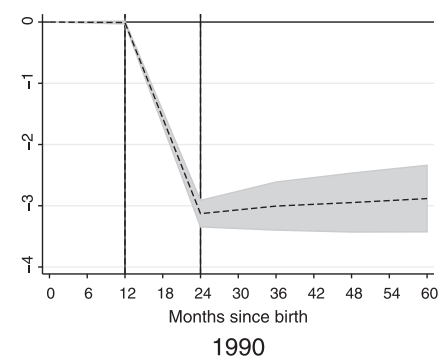

$(\mathbf{g})$

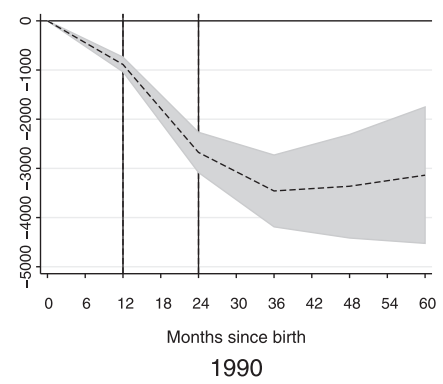

(b)

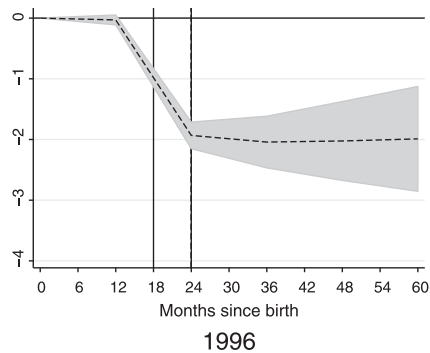

(e) 2. Months Unemployed

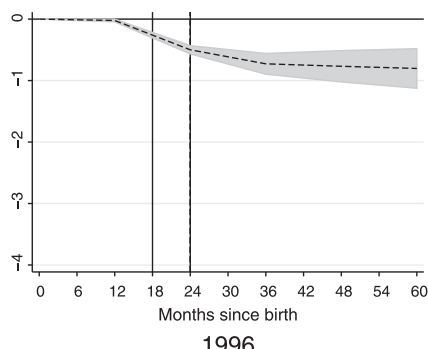

(h) 3. Cumulative Earnings

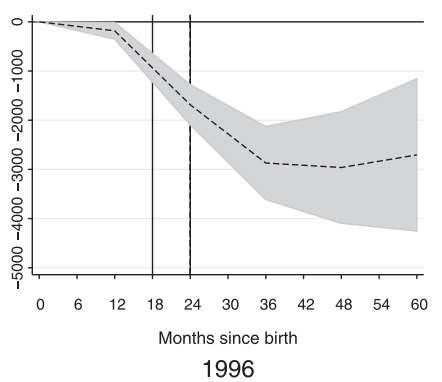

(c)

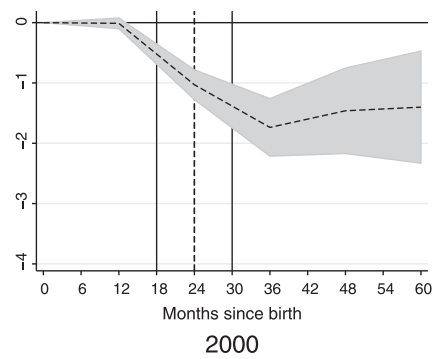

(f)

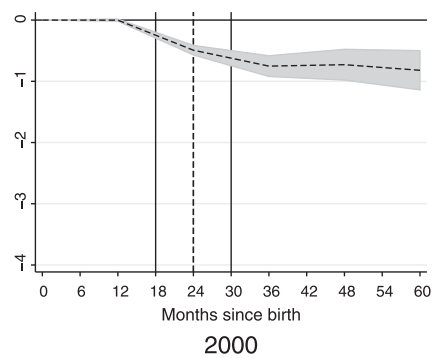

(i)

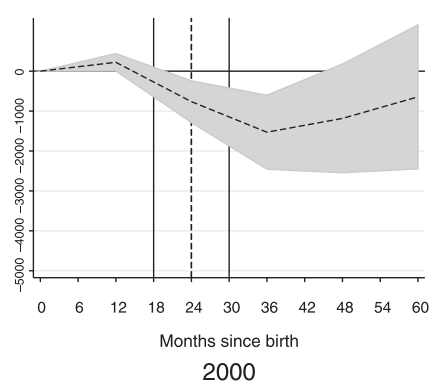

FIGURE 5

Reduced form effects on cumulative outcomes.

Notes: This figure plots DID-RD estimates (along with confidence intervals) for the impacts of the reforms on cumulative outcomes by months since the child's birth. The samples include all mothers who gave birth at parity one between May 1 and August 30. Estimates come from regressions that compare outcomes between the cohort exposed to the more generous regime and the cohort exposed to the less generous regime relative to the outcomes of pre- and post-July mothers in the pre-reform year. Regressions control for age at birth, and the following indicators for mothers' labour market performance measured 12 months before the child's birth: tenure, experience, months of unemployment, cumulative income, daily wages, and indicators for industry, region and white-collar occupation. Regressions also control for the unemployment rates in the region of pre-birth employment at the end of the job protection and benefit periods

during the period where the two groups face different PL regulations. Namely, we do not observe further losses in labour market experience after both groups have exhausted their PL leaves. Overall, as reported in the first row of Table 3 we see that the 1990 reform reduces work experience by 3.2 months, the 1996 reform by 2 months, and the 2000 reform reduces experience by 1.4 months. Interestingly, while extension of leave regulations significantly prolonged the time until return-to-work, the loss in work experience was much smaller. 
TABLE 3

The causal effects of the reforms on cumulative outcomes in year 5 after child's birth

\begin{tabular}{lccc}
\hline Year of reform & $\begin{array}{c}1990 \\
(1)\end{array}$ & $\begin{array}{c}1996 \\
(2)\end{array}$ & $\begin{array}{c}2000 \\
(3)\end{array}$ \\
\hline Months in employment & $-3.224^{* *}$ & $-1.989^{* *}$ & $-1.4^{* *}$ \\
& $(0.441)$ & $(0.441)$ & $(0.474)$ \\
Months unemployed & 17.528 & 20.432 & 19.167 \\
& $-2.882^{* *}$ & $-0.802^{* *}$ & $-0.819^{* *}$ \\
Cumulative earned income & 0.278 & 0.164 & 0.163 \\
& 7.329 & 3.398 & 3.029 \\
& $-3138^{* *}$ & $-2706^{* *}$ & -643.1 \\
Number of observations & $(707)$ & $(790)$ & $(920.4)$ \\
& 25,468 & 32,731 & 31,472 \\
\end{tabular}

Notes: This table reports DID-RD estimates for the impacts of the 1990, 1996, and 2000 reforms on mother's cumulative outcomes observed in year 5 after the child's birth. The samples include all mothers who gave birth at parity one between May 1st and August 30th. Regressions compare differences in outcomes between the cohort exposed to the more generous regime and the cohort exposed to the less generous regime relative to the outcomes of pre- and post-July mothers who gave birth in the year preceding the reform. Estimates come from regressions that control for age at birth, and the following indicators for mothers' labour market performance measured 12 months before the child's birth: tenure, experience, months of unemployment, cumulative income, daily wages, and indicators for industry, region, and whitecollar occupation. Regressions also control for the unemployment rates in the region of pre-birth employment at the end of the job protection and benefit periods. Robust standard errors are reported in parentheses. Means of the comparison group (i.e., the group with access to the less generous regime) are reported in italics. *Significant at 5\%, **Significant at $1 \%$.

Why does not extended parental leave crowd out work experience one-for-one? We find that mothers under the less generous PL regimes return-to-work earlier but have less stable employment immediately after birth. Moreover, mothers under the less generous regimes compensate it with higher participation rates in other social insurance programmes, such as unemployment insurance, which also provides income replacement while not employed 19 Indeed, as seen in the second row of Figure 5 and summarized in Table 3 mothers who face the less generous PL regimes claim about 3 additional months of unemployment benefits in 1990 and almost one additional month in 1996 and 2000 relative to their counterparts in the more generous regimes. The gap in months unemployed between the two groups of mothers is generated during the first 36 months after the child's birth.

The last row of Figure 5 plots annual differences in cumulative earnings from work since the child's birth. The extended time on leave generated an earnings loss of 3100 and $2700 €$ for mothers who gave birth in the more generous regimes of 1990 and 1996. Interestingly, the gap in cumulative earnings from work between mothers in the less and the more generous leave regimes is entirely generated in the first 36 months after the child's birth 20 From then on, we do not observe any further increases in the gap in cumulative earnings. This finding is important as it suggests that while mothers in the more generous leave regimes suffer from a permanent income loss, this loss is totally generated by forgone earnings during the leave period with no further consequences on earnings capacity once they return-to-work.

We next turn to examine labour market outcomes observed in the fifth year after the child's birth. A key potential challenge in examining post-birth labour market performance is differential

19. Unemployment insurance is conditional on work experience prior to claiming benefits and treats PL take-up as work experience. Most of the mothers in our sample are eligible for unemployment benefit receipt.

20. Note that income losses in the 2000 reform are smaller as mothers could work while still receiving benefits starting from month 18 after the child's birth. Still we do see losses for mothers giving birth at parities higher than one as reported in Appendix Table A.3 
selection into employment among pre- and post-July mothers. We examine this issue in Appendix A.1 where we compare employment rates of pre- and post-July mothers by year since the child's birth. We conduct a selection analysis that consists of measuring whether employed mothers have different pre-birth characteristics in the treated and control group. The selection analysis leads us to conclude that not only employment rates of pre- and post- July mothers are similar once both groups have exhausted their PL provisions, but we can also assure that pre- and post- July mothers who are employed come from the same part of the earnings potential distribution starting from year 3 after child's birth 21 These two findings are important as they imply that a comparison of labour market outcomes between pre- and post-July mothers in the medium and in the long run is unlikely to be confounded by differences in characteristics across the groups.

Table 4 reports $D I D-R D$ estimates of the impacts of the three reforms on labour market outcomes observed in the fifth year after the child's birth. As seen already in Figure A.1 we find no differences in employment rates between pre- and post-July mothers. We therefore conclude that despite the fact that more generous leave regimes delay mothers' return-to-work, they do not have any detrimental impact on employment in the medium-run 22

We also do not find any significant differences in the likelihood that mothers are still working for their pre-birth employer 5 years after birth. If anything, mothers in the more generous PL regimes are more likely to continue working for their pre-birth employer 5 years after the child's birth (for mothers giving birth at parity one). Another interesting finding is that despite the negative impacts of the extended leave regimes on work experience, tenure with current employer is not significantly affected by the reforms 23 Furthermore, we find that labour market attachment as reflected by the number of months worked in year 5 after the child's birth is unaffected by the longer leaves taken by mothers in the more generous regimes. More interestingly, we see that despite the delay in return-to-work and loss in work experience, there are no detrimental impacts on daily earnings (neither when we examine all mothers nor when we condition on employment) or on annual earnings 24 This last finding suggests that longer leaves do not generate significant losses in human capital. This will be relevant in the behavioural model presented below.

21. While we do not find any differences in observed characteristics, there could of course be differences in unobserved characteristics. We cannot entirely rule out this possibility, even though the lack of any differences in observables hints that the presence of large differences in unobservables is very unlikely, especially if these unobservables are correlated with the observed covariates.

22. As noted above, we find a lower return-to-work rate within the first 5 years after birth among mothers who gave birth in the more generous leave regime (treated group) relative to mothers who faced the less generous leave regime (comparison group). At the same time, we see that employment rates of both treated and comparison groups are similar at the end of year 5. These two findings can be reconciled by the fact that a larger share of mothers in the comparison group returned to work earlier but remained employed for only a few months end eventually left their jobs. That is, returning to work is not a permanent state for some mothers and indeed we see that some of them return-to-work only temporarily and leave again their jobs because they give birth again or because they find it difficult to combine childrearing and work. In fact, we find that employment rates and number of months worked during the calendar year are similar in treated and comparison groups starting from year 3 after birth even though more mothers in the comparison group returned to work. When we focus on mothers who return-to-work within the first 2 years after birth we see that comparison mothers who return-to-work within 24 months are more likely to work in the first two years after the child's birth (because they return earlier) but they are also less likely to remain employed afterwards. This finding also explains why delays in return-to-work are not one-to-one translated into differences in labour market experience observed in year 5 after birth.

23. Note that while we observe a marginally negative impact on tenure for the 2000 reform for mothers who give birth at parity one, we do not observe a negative impact for mothers who give birth at higher parities (see Appendix Table A.3 for estimates for mothers at parities greater than one).

24. We also examine labour market outcomes in the longer run, by looking at the effects of the 1990 and 1996 reforms in year 10 and 9 after birth, respectively. Results, not reported here but available upon request, show no significant differences in employment rates or earnings between pre- and post-July mothers. 
TABLE 4

The causal effects of the reforms on labour market outcomes in year 5 after child's birth

\begin{tabular}{|c|c|c|c|}
\hline Year of reform & $\begin{array}{c}1990 \\
(1)\end{array}$ & $\begin{array}{c}1996 \\
(2)\end{array}$ & $\begin{array}{c}2000 \\
(3)\end{array}$ \\
\hline Employed & $\begin{array}{c}0.002 \\
(0.013) \\
0.404\end{array}$ & $\begin{array}{c}0.000 \\
(0.014) \\
0.513\end{array}$ & $\begin{array}{c}0.009 \\
(0.015) \\
0.486\end{array}$ \\
\hline Working for pre-birth firm & $\begin{array}{c}0.061 * * \\
(0.020) \\
0.374\end{array}$ & $\begin{array}{c}0.025 \\
(0.019) \\
0.388\end{array}$ & $\begin{array}{c}-0.011 \\
(0.020) \\
0.400\end{array}$ \\
\hline Tenure with current employer & $\begin{array}{c}0.677 \\
(1.754) \\
48.683\end{array}$ & $\begin{array}{c}0.008 \\
(1.587) \\
50.367\end{array}$ & $\begin{array}{c}-3.475 \\
(1.836) \\
53.156\end{array}$ \\
\hline Months worked & $\begin{array}{c}-0.007 \\
(0.147) \\
5.033\end{array}$ & $\begin{array}{c}0.057 \\
(0.157) \\
6.281\end{array}$ & $\begin{array}{c}0.072 \\
(0.160) \\
6.064\end{array}$ \\
\hline Earnings per day worked & $\begin{array}{l}1.246 \\
(0.757) \\
41.309\end{array}$ & $\begin{array}{c}1.141 \\
(0.742) \\
43.624\end{array}$ & $\begin{array}{c}-0.837 \\
(0.825) \\
44.962\end{array}$ \\
\hline Earnings per calendar day & $\begin{array}{c}0.847 \\
(0.639) \\
16.691\end{array}$ & $\begin{array}{c}0.579 \\
(0.736) \\
22.377\end{array}$ & $\begin{array}{c}-0.303 \\
(0.754) \\
21.693\end{array}$ \\
\hline Annual income & $\begin{array}{c}239.7 \\
(225.2) \\
6977\end{array}$ & $\begin{array}{c}336.9 \\
(282.6) \\
9644\end{array}$ & $\begin{array}{c}-299.9 \\
(270.0) \\
9008\end{array}$ \\
\hline Number of observations & 21,507 & 21,146 & 18,345 \\
\hline
\end{tabular}

Notes: This table reports DID-RD estimates for the impacts of the 1990, 1996, and 2000 reforms on mother's labour market outcomes measured in year 5 after the child's birth. The samples include all mothers who gave birth at parity one between May 1st and August 30th. Regressions compare differences in outcomes between the cohort exposed to the more generous regime and the cohort exposed to the less generous regime relative to the outcomes of pre- and post-July mothers who gave birth in the year preceding the reform. Estimates come from regressions that control for age at birth, and the following indicators for mothers' labour market performance measured 12 months before the child's birth: tenure, experience, months of unemployment, cumulative income, daily wages, and indicators for industry, region, and white-collar occupation. Regressions also control for the unemployment rates in the region of pre-birth employment at the end of the job protection and benefit periods. Robust standard errors are reported in parentheses. Means of the comparison group (i.e., the group with access to the less generous regime) are reported in italics. *Significant at 5\%, $* *$ Significant at $1 \%$.

\section{BEHAVIOURAL FRAMEWORK}

This section lays out a structural search model to reproduce the empirical return to work patterns of mothers under different PL systems. The objective is to understand the differential roles played by job protection and cash benefits, two time-delimited policy instruments, in shaping mothers' return to work behaviour.

\subsection{A non-stationary search framework}

Mothers give birth in $t=0$ and quit their job earning them wage $w_{o}$, the pre-birth or protected wage, to go on PL25 While on PL, mothers engage in parenting or home production and gain value $c_{t}$ per period. We assume unobserved heterogeneity in $c_{t}$. For some time period $\tau_{o}$ mothers can return to their pre-birth job at wage $w_{o}$ and they receive cash benefits of $b$ for $\tau_{b}$ periods, where $b$ is a fixed cash amount equal for all mothers. Thus, high-wage mothers have a lower

25. The first 8 weeks after birth are not governed by parental leave but maternity insurance, which is mandated time off work by law. Therefore, mothers are only able to consider going back to work at the end of week 8 . In the model, we capture this fact by starting to compute reservation wages at $t=8$. We neglect this detail in the following exposition. 
replacement rate than low-wage mothers. While on leave, mothers engage in job search receiving a job offer with probability $\lambda$ per period, drawn from the wage offer distribution $F_{t}(w)$. A period in the model is a week. There are no search costs, jobs last forever, there is no on the job search, and job offers are assumed to be on a take-it-or-leave-it basis for mothers (no recall).

The value of $\mathrm{PL}$ at the end of period $t, V_{t}$, i.e. after rejecting any pending job offers, is given by the following Bellman equation 26

$$
V_{t}=\max \left\{\frac{w_{o}}{1-\beta}, c_{t}+b_{t}+\beta V_{t+1}+\beta \lambda \int_{0}^{\infty} \max \left[0, \frac{w}{1-\beta}-V_{t+1}\right] d F_{t+1}(w)\right\} \quad t \leq \tau_{o},
$$

where $\beta$ is the (weekly) discount factor and $b_{t}=b$ if $t \leq \tau_{b}$ and zero otherwise. If $t>\tau_{o}$ the outer max-operator drops out. Under the assumption of perfect foresight regarding the development of all the fundamental parameters, agents are able to solve this Bellman equation recursively.

Mothers decide in $t$ whether to accept job offer $w$ by comparing the value of accepting $w$ to the value of continuing search and the value of returning to the pre-birth job in $t$. Mothers can thus search for new jobs and still return to the pre-birth job in the same period if no suitable job offer has arrived. Since jobs last forever, equation (10 directly leads to the reservation wage $w_{t}^{*}$ as follows:

$$
w_{t}^{*}=\max \left\{w_{o},(1-\beta)\left(c_{t}+b_{t}\right)+\beta w_{t+1}^{*}+\beta \lambda \int_{w_{t+1}^{*}}^{\infty}\left(x-w_{t+1}^{*}\right) d F_{t+1}(x)\right\} \quad t \leq \tau_{o},
$$

where the max-operator is dropped for $t>\tau_{o}$. A mother will accept any new wage offer that pays more than the reservation wage. The optimal time of returning to the pre-birth job is the point in time where $V_{t} \leq w_{o} /(1-\beta)$, or equivalently, $w_{t}^{*} \leq w_{o}$, i.e. the continuation value of search at the end of period $t$ is less than returning to the pre-birth job. This is proven for the general case in continuous time in Frijters and Van der Klaauw 2006 27 They further show that there is a unique optimal time of return if the reservation wage is monotonically decreasing over time 28

We build on the job search model with non-participation laid out in Frijters and Van der Klaauw 2006, 29 Their model extends Van den Berg 1990)'s nonstationary job search model by allowing non-participation, an absorbing state. We extend their framework by time-delimiting the outside option, which in our case is return to the pre-birth

26. A note on the timing. The mother enters period $t$ with a job offer $w$ with probability $\lambda$. She then compares the present discounted value of the new job (if available) to the value of returning to the pre-birth job and the value of staying on PL. If she decides to stay on PL she collects $c_{t}+b_{t}$ in $t$ and enters period $t+1$ facing the same decision problem. If she decides to accept offer $w$ or return to pre-birth job, she starts collecting $w$ or $w_{o}$ in $t$ but does not receive $c_{t}$ or $b_{t}$ in $t$.

27. In their model, the outside option is not time delimited. However, their Theorem 1 and Condition 5 in the Appendix can be extended to include this case. One then needs to differentiate between points in time before the outside option has expired and after.

28. We compute the optimal time of return to pre-birth job by checking when the reservation wage is less or equal to the pre-birth wage. We thus rely on their result regarding uniqueness of time of return and therefore need all reservation wages to be declining. This restricts the parameter space of the model since increasing reservation wages are not ruled out per se. We run the estimation unconstrained and check whether parameters at the solution lead to declining reservation wages for all mothers in the sample.

29. A search model is well suited to study the two policy instruments since job protection has a clear value and interpretation only in a market with frictions. In a neoclassical labour market, mothers would not benefit from job protection since they would offer their labour productivity competitively at any point in time if the value of working would exceed the value of not working. Also note that we see a constant flow of mothers accepting new job offers in the data, pointing to the fact that some fraction of mothers are actively searching for jobs (i.e. those for which search costs are lower than expected gains from search). 
job. As discussed above, the PL rules in Austria provide mothers with the right to return to their pre-birth jobs for some time after birth. While on parental leave, mothers can engage in search for a new job while retaining their right to return to the pre-birth job at any time, and are entitled to cash benefit payments. Since both the duration of job protection and the duration of cash benefit payments are time delimited, our model features interaction effects between the two policy instruments. This is a novel feature of our model which, to the best of our knowledge, has not been previously studied.

Since returning to the pre-birth job is an absorbing state, the reservation wage is not defined for periods after the optimal time of return. Further, the reservation wage is non-stationary due to the time dependency of cash benefits, job protection, home production, and the wage offer distribution. Equation (2) shows clearly that the reservation wage and thus also the optimal time of returning to the pre-birth job depend on $\tau_{b}$ and $\tau_{o}$, the duration of cash benefits and job protection, respectively. The roles played by these two policy instruments is explored next.

\subsection{Cash benefits, job protection, and the reservation wage}

Return-to-work behaviour of mothers is affected differentially by the two policy instruments, duration of cash benefit transfers, and duration of job protection. Figure 6 compares the impact of alternative PL-systems on the evolution of the reservation wag 30: no PL provisions (panel (a)); 1 year of benefits but no job protection (panel (b)); 1 year of job protection but no benefits (panel (c)); 1 year of both cash benefits and job protection (panel (d)); 1 year of benefits and 2 years of job protection (panel (e)); and 2 years of benefits and 1 year of job protection (panel (f)).

A comparison of panels (a) and (b) shows that, starting from a situation without any PL provisions, the introduction of cash benefits increases the reservation wage significantly during the covered period. Extending PL benefit duration, increasing $\tau_{b}$, leads mothers to delay returnto-work because their reservation wage is higher.

To see the impact of introducing job protection, compare panels (a) and (c). The reservation wage of the mother in the system with job protection is initially above the pre-birth wage. The reservation wage then decreases since the value of home production decreases with time since birth. Eventually, the mother returns to work for sure in month 7 since her reservation wage hits the floor of 282.9 Euros per week - the wage she gets in her pre-birth job. This speeds up expected time of return-to-work since returning is now certain within 7 months given job protection. In contrast, this mother may still be on PL after 5 years in the baseline situation. Note that the reservation wage during PL in the system with job protection is higher, delaying return to a new employer. The combination of these two effects implies that job protection may either speed up or delay return-to-work.

We have explored more generally how duration of job protection affects average return-towork. We find that extending job protection initially speeds up return to work, as mothers who want to stay home for some time before returning to work start to take advantage of their right to return to the pre-birth job (which is why they discard their right if protection is too short). After some point (about 2 years), extending protection further starts to delay average return-to-work, due to higher reservation wages and the delay in return of the mothers who exhaust their protected period. Note further that cash benefits affect return-to-work behaviour of all mothers. In contrast, job protection does not affect low-wage or high-home production mothers, since their reservation wage is above the pre-birth wage.

30. To illustrate the impact of these PL regimes on reservation wages, we pick one example mother (a particular vector of characteristics, pre-birth wage and sample draw from home production distribution, see notes to Figure 6, and use the parameter estimates of the next subsection to calculate behavioural responses. 
(a) No job protection/cash benefits

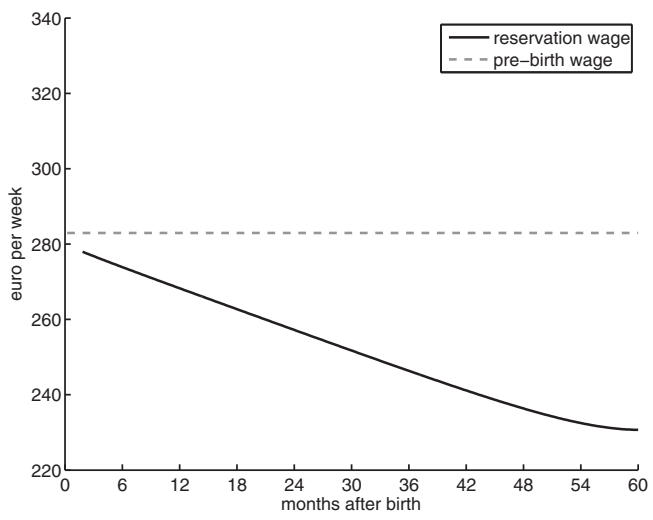

(c) Only job protection

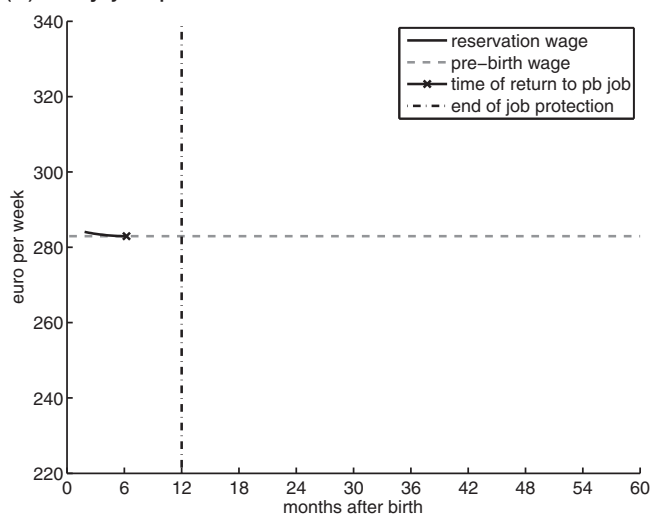

(e) $\mathrm{BD}<\mathrm{JPD}$

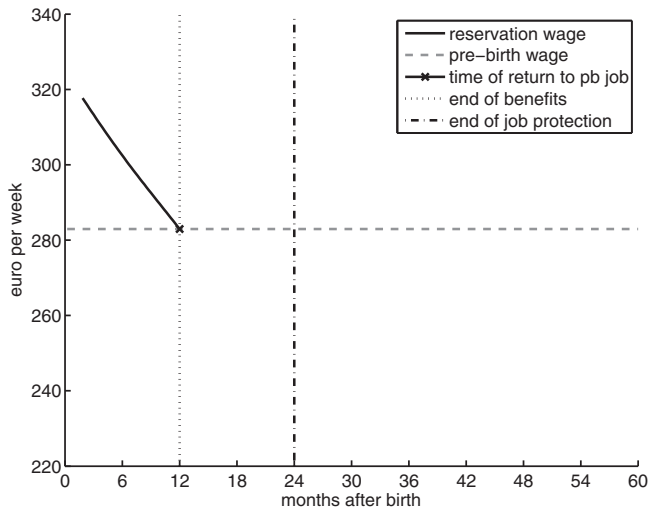

(b) Only cash benefits

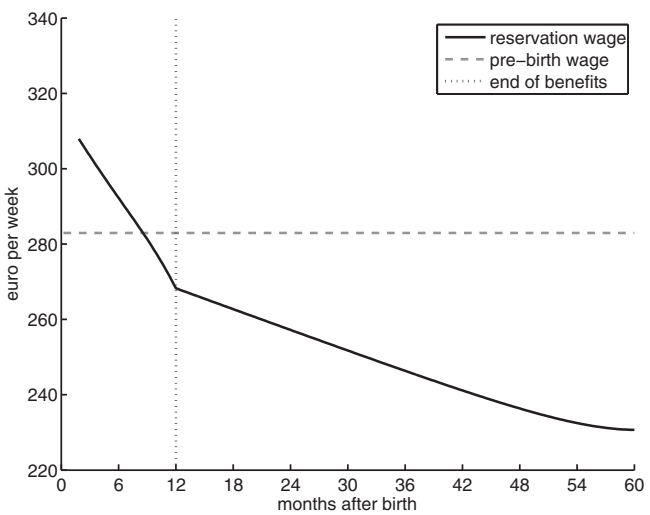

(d) $\mathrm{BD}=\mathrm{JPD}$

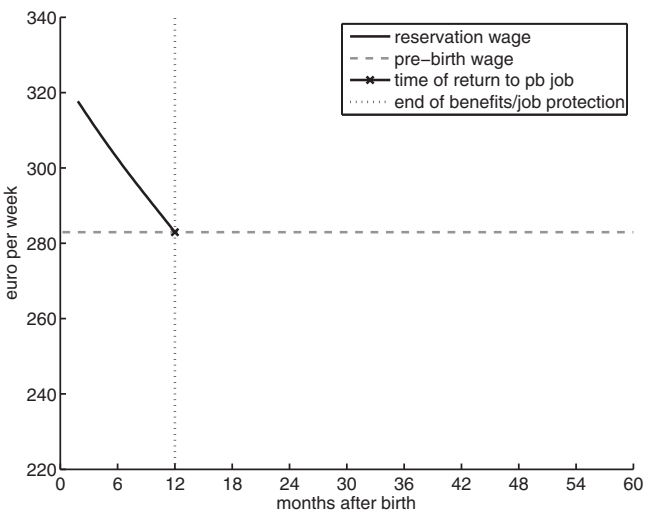

(f) $\mathrm{BD}>\mathrm{JPD}$

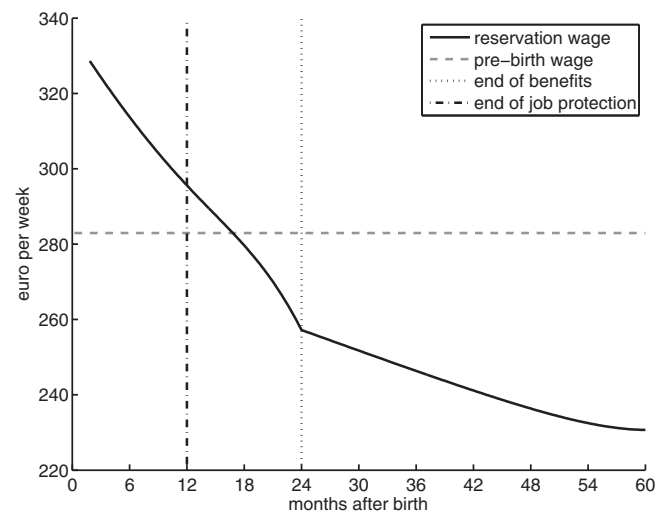

FIGURE 6

Reservation wages under different policyregimes.

Note: $\mathrm{BD}=$ benefit duration, JPD = job protection duration. The figures plot reservation wages for a selected Austrian mother with a pre-birth wage of 282.9 euro/week (median) and an initial value of home production of 328.7 euro/week (about 1st quintile of home-productivity distribution). Parameter values for the model are taken from Table 5 In this example, the other covariates are fixed at: blue-collar worker, age 27 years, region west of Austria, working in manufacturing. This gives a job offer probability of 0.029 , mean home production of 904.1 (the example reflects one selected draw from this distribution) and median wage offer of 283.0 euro/week 
Panels (d)-(f) of Figure 6 show how the two policy instruments interact. The same mother that returned to her pre-birth job in month 7 with only job protection (panel (c)) is induced to exhaust the protected period in the system that also provides benefits (panel (d)) 31 Extending job protection beyond the end of benefit payments (panel (e)) does not affect her optimal time of returning after 12 months. This is because the expected value of search is low (cf. panel (a)) and the opportunity costs due to the relatively high pre-birth wage are substantial. In panel (f), where benefits end after job protection, the mother does not make use of the right to return to her pre-birth employer. This means that the expected present discounted value of PL exceeds the value of the pre-birth job at all times during job protection.

\subsection{Structural estimation}

In the following, we discuss how we estimate the above model structurally. We assume that wage offers are generated by

$$
\log w_{i, t}=\mu_{i, t}+\epsilon_{w, i},
$$

where $\epsilon_{w} \sim N\left(0, \sigma_{w}^{2}\right)$ and $i$ denotes individual $i$. The mean of this distribution is given by $\mu_{i, t}=$ $x_{i}^{\prime} \theta_{w}-\gamma t$ where $x_{i}$ is a vector of covariates, and $\gamma$ measures the evolution of the mean of the $(\log )$ wage offer distribution with time since birth $t$. Accepted post-birth wages are subject to measurement error, since structural estimation of job search models is highly sensitive to outliers otherwise (see Wolpin 1987) for an excellent treatment). Assume that observed wage $\hat{w}_{i}$ is generated by

$$
\log \hat{w}_{i}=\log \tilde{w}_{i}+u_{i}, \quad u \sim N\left(0, \sigma_{u}^{2}\right)
$$

where $\tilde{w}_{i}$ is the true accepted wage, and $u$ is measurement error. In our data, the measurement error also captures the discrepancy between the hourly wage and daily earnings, e.g. changes in hours of work.

We assume that the value of home production varies across individuals and over time and takes the following form.

$$
c_{i, t}=x_{i}^{\prime} \theta_{c}-\alpha t+\epsilon_{c, i},
$$

where $\epsilon_{c} \sim N\left(0, \sigma_{c}^{2}\right)$. The stochastic component $\epsilon_{c}$ introduces unobserved heterogeneity into the model. The offer arrival probability is assumed to be constant over time but is allowed to vary across individuals in line with their observed characteristics $x_{i}$.

$$
\lambda_{i}=\frac{\exp \left[x_{i}^{\prime} \theta_{\lambda}\right]}{1+\exp \left[x_{i}^{\prime} \theta_{\lambda}\right]}
$$

The logistic specification ensures that $\lambda_{i}$ has support $(0,1)$. Cash benefit payments in the model take the following representation.

$$
b_{t}= \begin{cases}b \cdot k & \text { if } t \leq \tau_{b} \\ 0 & \text { else }\end{cases}
$$

where $b=101$ euro per week-the actual flat cash benefit mothers received in the 1996-2000 period. The reduced form results of Section 4 showed that the end of PL benefits induces many mothers to return to work.

31. Note that this is not a knife-edge case. A positive mass of mothers, i.e. a range of values of the home production distribution, return at benefit/job protection end. This range is calculated in the Appendix, where we derive the likelihood contribution of mothers in the sample who return to pre-birth jobs. 
We introduce the parameter $k$ to appropriately account for the utility value of PL benefits 32 This parameter captures the fact that one euro of benefits may be worth more-in utility terms - than one euro of wages, for instance, because the marginal utility of consumption is higher when the mother is not working than when she is working. This may arise if there are complementarities in consumption and leisure and individuals do not save (as often assumed in the job search framework). As an example, think about the stress associated with combining child care and work (driving time to nearest child care facility, time constraints due to opening hours, sickness of the child, etc.). In such a situation, mothers place a higher value on 400 euro of monthly PL benefits than on a 400 euro wage associated with stressful working.

There are three possible outcomes in our data. First, mothers can still be on leave at the end of our observation period $T=260$ ( 5 years). Second, mothers can be observed to return to their prebirth employer at $t_{i} \leq \tau_{o}$. Third, mothers can start working at a new job at $t_{i}$ for observed wage $\hat{w}$. The likelihood contributions for these three cases are derived in the Appendix. We use simulated maximum likelihood to integrate out unobserved heterogeneity. The model is computationally demanding, since we have to solve the whole reservation wage paths many times for every observation. To simplify computation of these paths, we assume that all parameters of the model are stationary after $T$.

We now turn to discussing identification. The home production component and discount rate of equation (2) are not separately identified with unobserved heterogeneity without observing reservation wages (see Flinn and Heckman 1982) and Frijters and Van der Klaauw 2006). Therefore, we fix the subjective discount rate $\beta$ to 0.999 , a value that implies an annual discount rate of 0.95 . We also check the sensitivity of our results to different values of $\beta$. To get identification of the wage offer distribution and job offer arrival rate, we follow the common approach in the literature and assume a log normal distribution with a time-dependent mean and constant variance for wage offers and a time invariant job offer arrival rate 33 Accepted wages identify the distribution and duration dependence of the wage offer distribution while the durations to new jobs identify the job offer arrival rate. The durations until return to pre-birth job identify the home production distribution and duration dependency under the assumption that the distribution is normal. This results from the fact that the reservation wage at the time of return has to be equal to the pre-birth wage, as discussed above. The benefit multiplier is identified separately from the home production components as the duration of benefits in the PL system we use to estimate the model was one and a half years while the duration of job protection was 2 years. Mothers who return before and after the end of benefits payments therefore provide separate identification of these parameters.

\subsection{Results}

We estimate the parameters of the non-stationary job search model outlined above structurally from our Austrian data set. From the data we know the exact time of birth, the pre- and postbirth daily earnings, the duration of PL (measured as time to first job after birth) and whether mothers return to their pre-birth jobs. After dropping mothers with missing values in some of the covariates, trimming the top and bottom $1 \%$ of the wage distribution and dropping mothers

32. In an earlier version of the article we used the most basic search model with $k=1$ to explain return-to-work behaviour of mothers. While the importance of benefits was a bit underestimated, the model was still able to replicate all the reforms qualitatively. Results are not critically dependent on this parameter, but the fit markedly improves.

33. Friiters and Van der Klaauw 2006 allow for non-parametric time dependency in the job offer arrival rate, which is possible with their data since they observe reservation wages. However, they do not find duration dependence in the arrival rate to be significantly different from zero. 
returning to pre-birth jobs after the end of job protection (about $12 \%$ of the sample) 34 , we end up with 99,400 mothers who gave birth between July 1996 and June 2000. These mothers are entitled to 1.5 years of cash benefits and 2 years of job protection. We estimate the model on a random subsample of 19,685 (about 20\%) mothers to reduce the computational burden of the estimation 35

Table 5 shows the estimation results of the structural model 36 Home production is estimated to have a high mean, a strong dispersion and is decreasing over time 37 Mothers' time with the child is more important the younger the child is. The high dispersion reflects significant unobserved heterogeneity in home production (and partners' earnings, which are not in our data) 38 The value of home production is increasing in the pre-birth wage. This finding is consistent with Guryan et al. (2008), who show that highly educated mothers spend more time with their children and Gould and Simhon 2011), who show that highly educated parents are more effective in enhancing children's human capital. Conditional on the wage, home production is lower for white-collar workers, which may be due to career continuity being more important for this group of women. We show age profiles of predicted home production, offer probability and wage offers for the base category in appendix Figure A.2

The weekly probability of receiving a job offer decreases with age quite dramatically from about $6 \%$ for young Austrian blue-collar mothers (age of 20 years) to less than $2 \%$ at the age of 40 years (see appendix Figure A.2 for a full age profile). Blue-collar women are more likely to receive wage offers than white-collar women and immigrant women are also more likely to be offered a job than Austrian citizens. Also, job offers arrive somewhat more frequently in Vienna and Southeast Austria.

The log-normal wage offer distribution is centered quite closely around the pre-birth wage with a dispersion of $\hat{\sigma}_{w}=0.028$. The dispersion of the measurement error distribution is larger, with $\hat{\sigma}_{u}=0.849$. The fact that measurement error has a strong variance reflects primarily changes in hours worked (which we cannot observe in our data), changes in working arrangements due to the presence of a child, and also genuine measurement error. The fact that the dispersion in wage offers is small is because the wage offer distribution varies with the pre-birth wage. This is a unique feature of our data. Structural search models are often estimated without knowledge of the wage at the previous job. The wage offer dispersion then confounds match quality with unobserved productivity.

The mean of the estimated wage offers is slightly increasing over time, but the effect is quantitatively small. This is surprising as one might expect leave-taking is associated with loss of human capital. Notice, however, that our specification - that allows for a shift in the mean of the wage offer distribution over time-is a reduced form for at least three potentially

34. In the model, mothers cannot return to pre-birth jobs after job protection has expired. Thus, these mothers have a likelihood contribution of zero (or negative infinity in $\log s$ ). Their behaviour therefore cannot be explained within the model.

35. Running the Fortran OpenMPI programme (BHHH optimization algorithm) took about 2 days occupying 128 cores on the University of Zurich's Schrödinger supercomputer. We ran the optimization programme with different starting values and converged to the same optimum.

36. The log likelihood function is locally concave. Starting from different initial values did not change the results. Reservation wages have to be decreasing to get a unique optimal time of return to pre-birth job. We therefore check whether the estimated parameters lead to this outcome, which is the case. Unconstrained estimation of this model allows us to rely on the usual asymptotics when computing the standard errors.

37. We re-scale home production (/100) to have estimates with similar magnitudes for numerical reasons. Thus $\sigma_{c}$ and all parameter estimates determining $\mu_{c}$ have to be multiplied by 100 .

38. Friiters and Van der Klaauw 2006) allow for unobserved heterogeneity in wage offers and the offer arrival rate. Since we do not observe reservation wages, this is not possible here, and we would argue that in the case of mothers' return to work after birth, unobserved heterogeneity is mainly important in the outside option. 
REVIEW OF ECONOMIC STUDIES

TABLE 5

Estimation results of the structural model

\begin{tabular}{lclc}
\hline$\alpha$ & 3.63295 & $\sigma_{u}$ & 0.84897 \\
depr. of home prod. value & $(0.26705)$ & measurement error dist. std.dev. & $(0.00661)$ \\
$\sigma_{c}$ & 5.47055 & $\gamma$ & 0.00009 \\
home prod. dist. std.dev. & $(0.39781)$ & wage offer dist. decline & $(0.00002)$ \\
$\sigma_{w}$ & 0.02725 & $k$ & 4.58990 \\
wage offer dist. std.dev. & $(0.00224)$ & benefit multiplier & $(0.33642)$ \\
\hline
\end{tabular}

\begin{tabular}{|c|c|c|c|}
\hline & Home prod. $\left(\mu_{c}\right)$ & Offer prob. $(\lambda)$ & Wage offers $\left(\mu_{w}\right)$ \\
\hline Constant & $\begin{array}{r}-3.18009 \\
(0.94391)\end{array}$ & $\begin{array}{r}-1.98386 \\
(0.61386)\end{array}$ & $\begin{array}{c}0.25243 \\
(0.03034)\end{array}$ \\
\hline Age $(/ 10)$ & $\begin{array}{l}3.34193 \\
(0.68793)\end{array}$ & $\begin{array}{c}-1.18975 \\
(0.44140)\end{array}$ & $\begin{array}{c}-0.05750 \\
(0.01797)\end{array}$ \\
\hline $\mathrm{Age}^{2}$ & $\begin{array}{r}-0.50976 \\
(0.12226)\end{array}$ & $\begin{array}{c}0.10274 \\
(0.07922)\end{array}$ & $\begin{array}{c}0.01116 \\
(0.00325)\end{array}$ \\
\hline Foreign & $\begin{array}{r}-1.58410 \\
(0.15813)\end{array}$ & $\begin{array}{c}0.15873 \\
(0.09302)\end{array}$ & $\begin{array}{r}-0.00906 \\
(0.00281)\end{array}$ \\
\hline White collar & $\begin{array}{r}-0.96114 \\
(0.12723)\end{array}$ & $\begin{array}{r}-0.22461 \\
(0.07142)\end{array}$ & $\begin{array}{c}0.00393 \\
(0.00230)\end{array}$ \\
\hline Pre-birth wage (log) & $\begin{array}{c}1.21957 \\
(0.08670)\end{array}$ & $\begin{array}{c}0.16837 \\
(0.05544)\end{array}$ & $\begin{array}{c}0.96839 \\
(0.00288)\end{array}$ \\
\hline \multicolumn{4}{|l|}{ Base region: West Austria } \\
\hline Southeast Austria & $\begin{array}{r}-1.57540 \\
(0.15122)\end{array}$ & $\begin{array}{c}0.27890 \\
(0.08654)\end{array}$ & $\begin{array}{r}-0.01501 \\
(0.00290)\end{array}$ \\
\hline Northeast Austria & $\begin{array}{r}-1.12400 \\
(0.12386)\end{array}$ & $\begin{array}{r}-0.00099 \\
(0.07206)\end{array}$ & $\begin{array}{c}-0.00854 \\
(0.00258)\end{array}$ \\
\hline Vienna & $\begin{array}{l}-2.22379 \\
(0.19000)\end{array}$ & $\begin{array}{l}0.27915 \\
(0.08101)\end{array}$ & $\begin{array}{c}-0.00996 \\
(0.00278)\end{array}$ \\
\hline Region missing & $\begin{array}{r}-0.75902 \\
(0.87473)\end{array}$ & $\begin{array}{r}-0.78634 \\
(0.30327)\end{array}$ & $\begin{array}{c}0.04304 \\
(0.03182)\end{array}$ \\
\hline \multicolumn{4}{|l|}{ Base industry: Manufacturing } \\
\hline Wholesale and retail trade & $\begin{array}{c}0.22218 \\
(0.12861)\end{array}$ & $\begin{array}{c}0.09747 \\
(0.08842)\end{array}$ & $\begin{array}{c}0.00293 \\
(0.00283)\end{array}$ \\
\hline Accommodation and food service & $\begin{array}{r}-0.80145 \\
(0.14679)\end{array}$ & $\begin{array}{c}0.27473 \\
(0.11136)\end{array}$ & $\begin{array}{c}0.00894 \\
(0.00360)\end{array}$ \\
\hline $\begin{array}{l}\text { Information, communication, } \\
\text { financial, and insurance services }\end{array}$ & $\begin{array}{r}-0.61659 \\
(0.20276)\end{array}$ & $\begin{array}{r}-0.42380 \\
(0.13939)\end{array}$ & $\begin{array}{c}0.00639 \\
(0.00476)\end{array}$ \\
\hline $\begin{array}{l}\text { Administrative and support service, } \\
\text { public administration }\end{array}$ & $\begin{array}{r}-4.45660 \\
(0.35454)\end{array}$ & $\begin{array}{r}-0.17414 \\
(0.10642)\end{array}$ & $\begin{array}{r}-0.00241 \\
(0.00332)\end{array}$ \\
\hline $\begin{array}{l}\text { Human health, social work, and } \\
\text { education }\end{array}$ & $\begin{array}{r}-1.80942 \\
(0.19528)\end{array}$ & $\begin{array}{c}0.02757 \\
(0.11204)\end{array}$ & $\begin{array}{r}-0.00263 \\
(0.00331)\end{array}$ \\
\hline $\begin{array}{l}\text { Professional, scientific, and } \\
\text { technical activities }\end{array}$ & $\begin{array}{r}-0.32077 \\
(0.19577)\end{array}$ & $\begin{array}{c}-0.31085 \\
(0.12905)\end{array}$ & $\begin{array}{c}0.01747 \\
(0.00520)\end{array}$ \\
\hline Other industry/missing & $\begin{array}{r}-0.72994 \\
(0.15181)\end{array}$ & $\begin{array}{c}0.17677 \\
(0.10743)\end{array}$ & $\begin{array}{r}-0.00105 \\
(0.00330)\end{array}$ \\
\hline $\begin{array}{l}\text { Number of observations } \\
\text { Log-likelihood }\end{array}$ & $\begin{array}{c}19,685 \\
-122,009\end{array}$ & & \\
\hline
\end{tabular}

Notes: Standard errors in parentheses. Subjective discount rate $(\beta)$ fixed to 0.998 (about $10 \%$ per year). Home production is divided by 100 for numerical stability. Region is determined by location of the plant where workers are employed. Industry categories are OENACE08 one digit groups as classified by Statistics Austria. About half a percent of observations have missing region while about $11 \%$ are in "other industry / missing" category.

important mechanisms: (i) depreciation of human capital, (ii) employers using information on the time since birth as a signal of effort/career commitment, or (iii) changes in desired hours of work. Depreciation of human capital and career commitment both imply that wage offers should decrease with time since birth. The third mechanism captures the idea that mothers may 
(a) Empirical return to work:

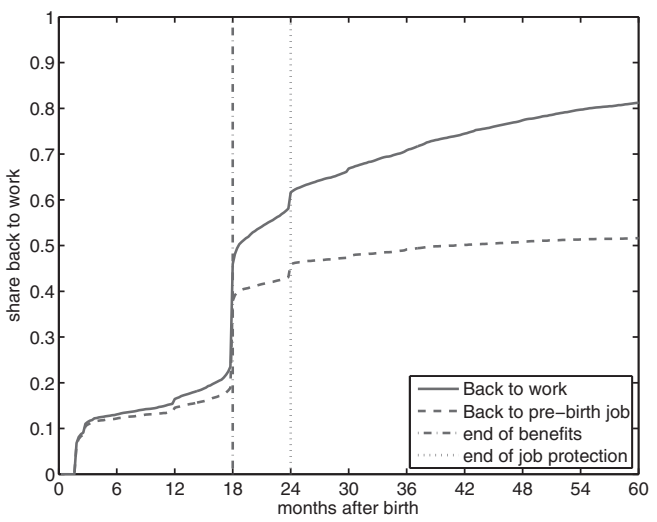

(b) Predicted return to work:

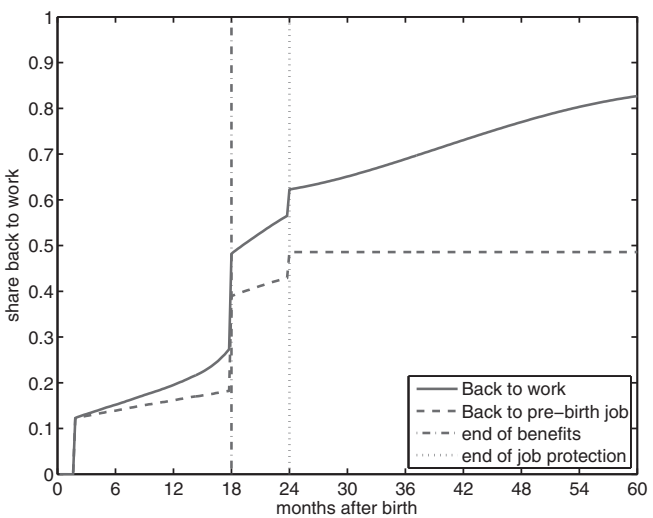

FIGURE 7

Empirical versus predicted return to work profile.

Note: Figures correspond to regime 3 (1996-2000) in Austria, where mothers were entitled to 18 months of benefits and 24 months of job protection. The predicted return to work profile is simulated with the parameters of Table 5 using a random sample of 1000 mothers

be willing to work more hours when returning later. Since our empirical specification captures the daily (rather than the hourly) wage, the empirically estimated wage offer distribution cannot capture the hours-component separately. In sum, the estimated specification captures the above three mechanisms in a reduced form. Our estimates indicate that the mean of the wage offer does not change strongly over time. This suggests that increases in desired hours offset the negative effect of human capital depreciation and signalling on wages. Note that this result is consistent with our reduced form results that find no evidence for human capital depreciation among the group of mothers exposed to longer leave regimes.

The parameter $k$ of 4.6 reflects the fact that cash benefits are important in enabling mothers to care for their young children. One interpretation is that the utility value of $1 \$$ of benefits is 4.6 times higher than the utility value of $1 \$$ of labour income. Notice, however, that we cannot separately identify the subjective discount factor, so we ultimately do not know whether mothers on average have lower home production and $k$ parameter but a higher subjective discount rate 39

Figure 7 compares the predicted return-to-work profile of the model to the empirically observed return-to-work profile. The model is able to match the aggregate return to work behaviour quite well. The most important features are replicated, mainly some share returning instantly and discrete jumps at the policy endpoints. Also, the proportion of mothers going to new jobs is well captured. The model predicts convex return to new jobs after the end of PL (2 years) which follows from decreasing home production values leading to lower reservation wages. The data, however, show a concave return to work pattern after 2 years, hinting at non-linearities in the depreciation of home production or decreasing home production but stable partner's earnings, which may be more dominant in later years after birth.

We evaluate the fit and suitability of the model further by predicting "out of sample" mothers' reaction to the Austrian PL reforms in the next section.

39. We estimated the same model with a fixed weekly discount factor of 0.999 and 0.997 (about $5 \%$ and $15 \%$ annual discount rate, respectively) and got similar results in most parameters except for the home production and benefit multiplier components. 


\section{PREDICTION AND POLICY}

The objective of this section is 2-fold. First, we assess to what extent the behavioural framework can predict the impact of the three Austrian PL reforms on mothers' return-to-work behaviour. Second, we use the behavioural framework to examine the impacts of three counterfactual policies: a regime with neither job protection nor benefits, a policy with just benefits, and a policy with just job protection. We contrast return-to-work profiles in these counterfactual systems to understand the relative importance of job protection and cash benefits in shaping return-to-work decisions.

\subsection{Predictive capability of structural estimates}

This subsection compares a series of simulated return-to-work profiles with their empirical counterparts. We do this by feeding the structural model above with the data underlying the reduced-form evidence of Section 13 40 This allows us to simulate how the three Austrian reforms affect return-to-work profiles. Figure 8 shows the reduced form return-to-work profiles in the left column and the simulated return-to-work profiles in the right column.

The 1990 reform extends job protection duration and benefit duration from 12 months to 24 months (Figure $8 \mathrm{~b}$ and b). This PL extension induces a delay in return to work that is concentrated in the second year after giving birth according to the empirical return-to-work profile. The simulated return to work profile replicates the delay in return to work in the second year after birth quite well. The key difference between the two sets of profiles occurs from the third year after birth onwards. While the empirical profile shows that fewer women have returned to work even after the end of the benefits and job protection period, the model predicts that the proportion of women having returned to work in the medium-run is not affected by the reform.

The 1996 reform reduces benefit duration from 24 months to 18 months keeping job protection at 24 months (Figure $8 \mathrm{k}$ and d). The reduction in benefit duration speeds up return to work primarily in months 18-24 according to the empirical return-to-work profile. The model captures this central feature of the data very well. Interestingly, the model also replicates quite well the $5 \%$ of women who remain on PL after benefits have run out but return to their pre-birth employer when job protection runs out.

The 2000 reform prolongs benefit duration from 18 months to 30 months again keeping job protection duration unchanged (Figure 8 and $\mathrm{f}$ ). The model captures the delay in return to work induced by the reform between month 18 (when benefits used to run out) and month 24 (when job protection runs out) well. The empirical return to work profile shows more women returning in month 18 after the extension of PL benefits than the simulated return to work profile. This is because some decisions to return to work were scheduled before the extension of PL benefits was enacted and because some mothers took advantage of the lift in the income ceiling and combined work and benefits starting from month 18 (i.e., after the reform became effective). Since we do not incorporate these features in the model, we do not interpret the differences between observed and predicted shares as a model-fitting failure. Moreover, the model also captures the medium-run difference in the share ever returning to work by month 60 quite well. The model does not work perfectly in predicting how many women return when job protection runs out.

40. The sample for the reforms are mothers giving birth in May-June (pre) versus July-August (post) in the reform years. For every observation, we draw 25 home production values and calculate reservation wage paths. From these, it is straightforward to get the hazards to new and old jobs and calculate the aggregate failure function. 
(a) 1990 reform (empirical)

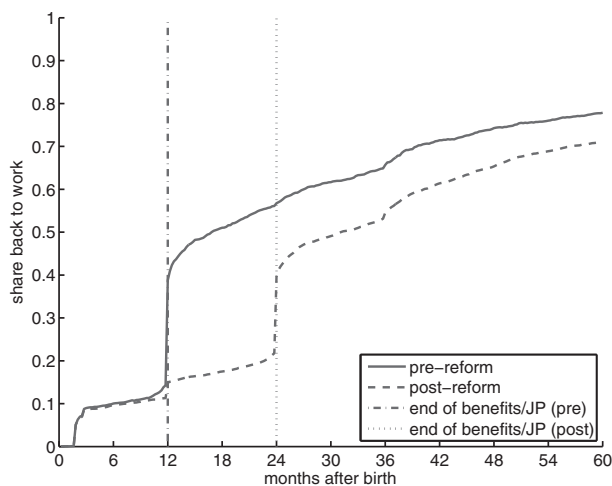

(c) 1996 reform (empirical)

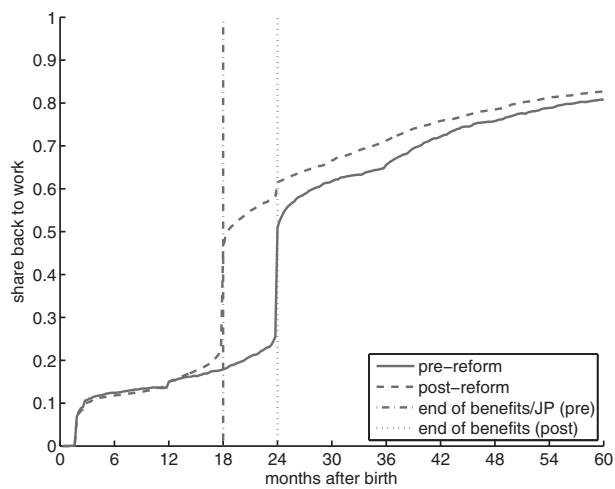

(e) 2000 reform (empirical)

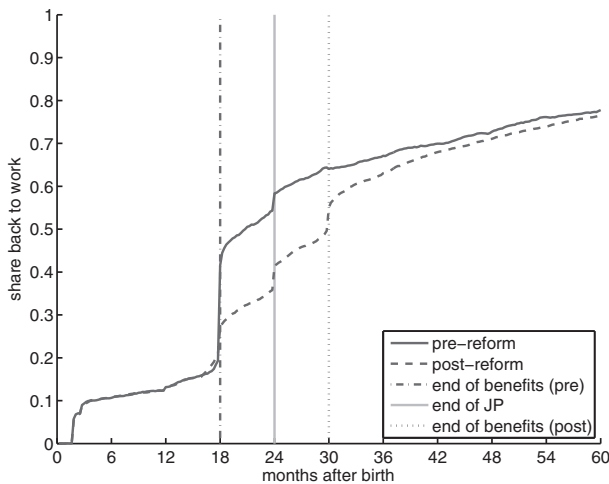

(b) 1990 reform (theoretical)

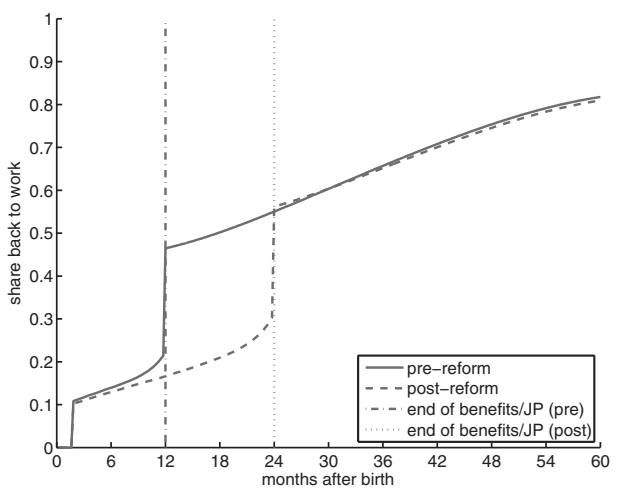

(d) 1996 reform (theoretical)

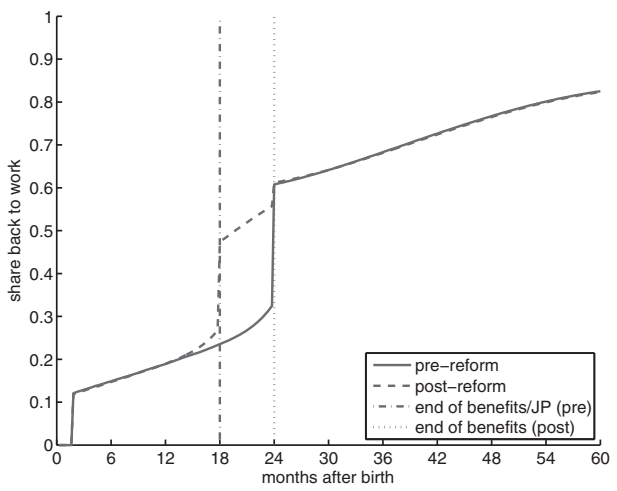

(f) 2000 reform (theoretical)

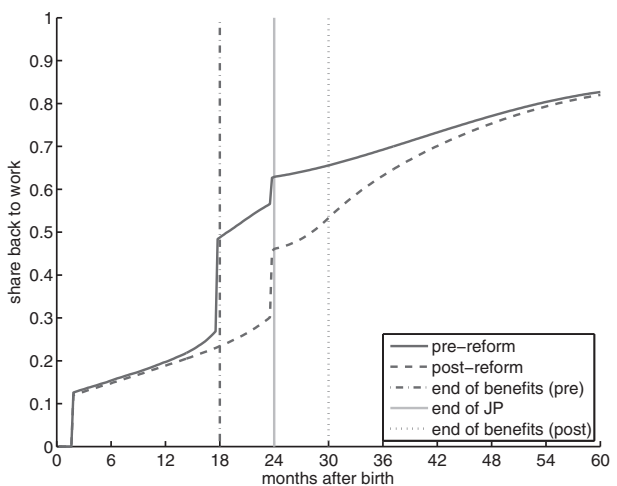

FIGURE 8

Empirical and simulated return-to-work profiles.

Notes: This figure shows the empirically observed and simulated return-to-work profiles (using the estimated model of the previous section) for three reforms of the Austrian PL system. (a) Shows the empirical profiles and (b) the simulated profiles for the 1990 reform that extended the duration of benefits and job protection from 12 months to 24 months, (c) shows the empirical and (d) shows the simulated profile for the 1996 reform that reduced benefit duration from 24 months to 18 months keeping job protection duration at 24 months, (e) shows the empirical, and (f) shows the simulated profile of the 2000 reform that extended benefit duration from 18 months to 30 months, again keeping job protection duration at 24 months. Empirically observed return-to-work profiles are corrected for seasonality (see Section 13 for 
The model puts this group at $20 \%$ of the total, whereas the reduced form puts it around $5 \%$. We see below that mothers were allowed to return to the pre-birth job even after job protection has ended. This discrepancy is due to informal arrangements between mothers and pre-birth employers that allow them to return to their pre-birth job after the end of the job protection period. Such informal arrangements are not captured in our model as they do not derive from the PL legislation.

How well does the model replicate return-to-same-employer profiles? Figure 9 displays the empirical (left) and simulated (right) share of women who have returned to the pre-birth employer as a function of time since birth. Results for the 1990 reform indicate that extending both job protection and benefit duration by 12 months induces an horizontal shift in the return-to-sameemployer profile. This feature is apparent in both the empirical and the simulated return to work profile. Extending the duration of job protection increases the share of mothers returning to the pre-birth employer. This is due to the declining value of home production: more women assess returning to their pre-birth employer as being attractive if they get to spend more time at home.

The 1996 reform, which reduces benefit duration by 6 months, induces some mothers who would have returned at 24 months to their pre-birth employer, to do so already at 18 months (i.e., when PL benefits run out). This fact is strong both in the empirical profile and is replicated in the simulated profile. In particular, the simulated profiles matches the medium-run proportion returning to the same employer very well.

The 2000 reform, which extends benefits by 12 months, induces most women who would have returned to their pre-birth employer after 18 months to do so after 24 months. There is a second group of women who would have returned to their pre-birth employer after 18 or 24 months but now do so after 30 months. Apparently, these women are able to negotiate a return to the same job even after job protection has run out. The simulated return-to-sameemployer profile mimics the shift from 18 months to 24 months but does not replicate the shift to 30 months since the model does not allow for delayed start dates. Yet, the model and the data agree that the 2000 reform reduces the share returning to work via their pre-birth employer by month 60 . The reduction in returns to the same employer is stronger in the model than in the data because the model does not allow for a return to the same employer after month 24 .

Overall, our behavioural framework is capable of replicating the four most important features of both the overall return-to-work profile and the return-to-same-employer profiles. First, both the empirical return-to-work profile and the simulated return-to-work profiles are discontinuous at the dates when benefits end. Second, the model manages to replicate the shares returning to the same employer and going to a new employer quite well. Third, changes to benefit or job protection duration affect return-to-work times more strongly through returns to the same employer than through search for a new job. Fourth, changes to benefit duration affect return-to-work more strongly in the period with a guaranteed option to return to the same employer than in other periods.

\subsection{The relative importance of job protection and cash benefits}

The structural model of job search suggests that mothers value time with their child very strongly right after birth. Moreover, the time period we analyse is one where there is very little child care for children below the age of 3 years. In this setting, a PL policy can support families of newborn children by generating opportunities for prolonged parental care immediately after birth while maintaining medium-run labour market attachment of parents. How well do job protection and 
(a) 1990 reform (empirical)

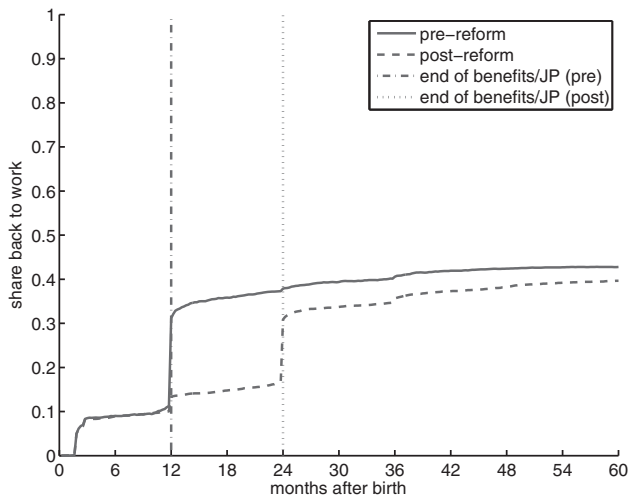

(c) 1996 reform (empirical)

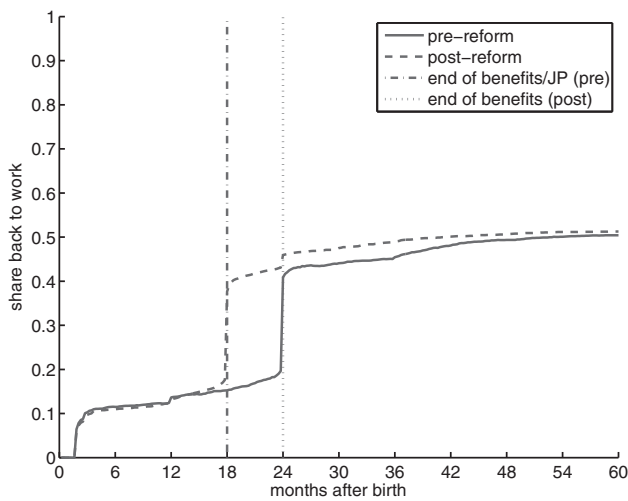

(e) 2000 reform (empirical)

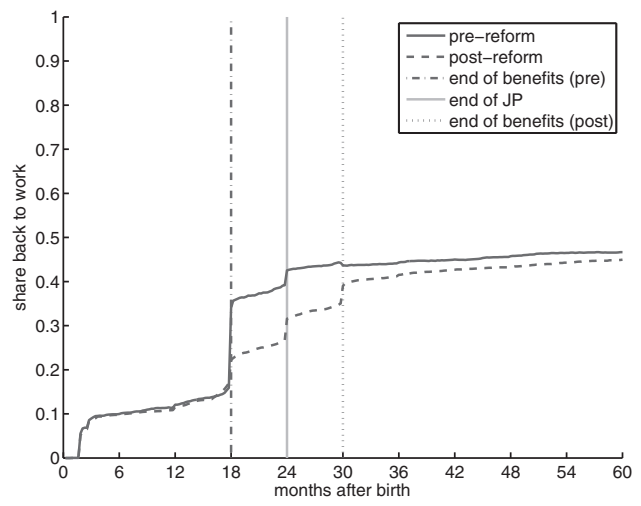

(b) 1990 reform (theoretical)

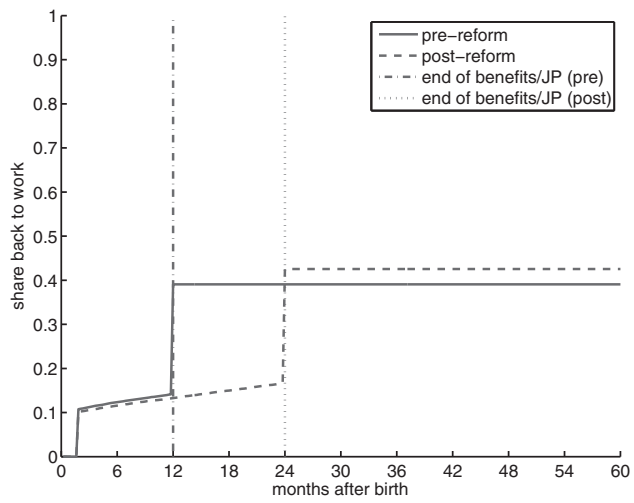

(d) 1996 reform (theoretical)

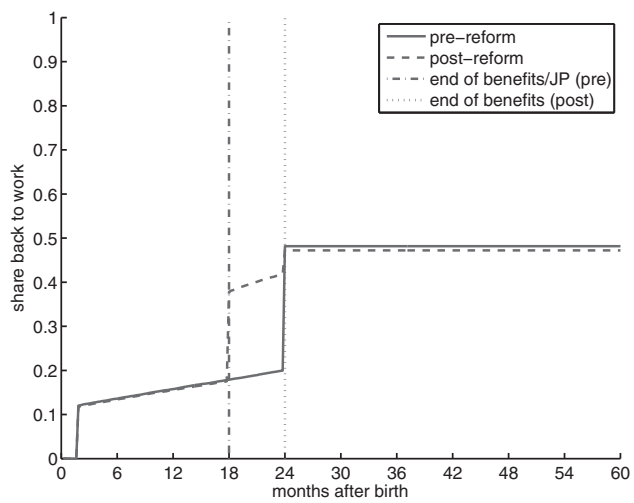

(f) 2000 reform (theoretical)

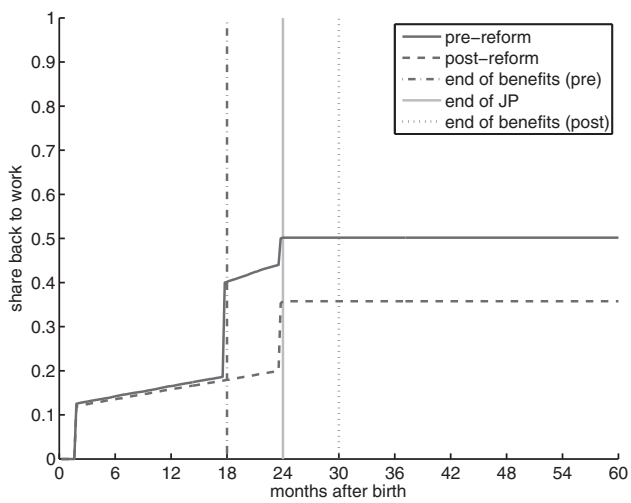

FIGURE 9

Empirical and simulated return-to-same employer profiles.

Notes: This figure shows empirical and calibrated return-to-same employer profiles for three reforms of the Austrian PL system. (a) shows the empirical and (b) the simulated profiles for the 1990 reform that extend the duration of benefits and job protection from 12 months to 24 months, (c) shows the empirical and (d) shows the simulated profile for the 1996 reform that reduces benefit duration from 24 months to 18 months keeping job protection duration at 24 months, (e) shows the empirical, and (f) shows the simulated profile from the 2000 reform that extends benefit duration from 18 months to 30 months, again keeping job protection duration at 24 months 

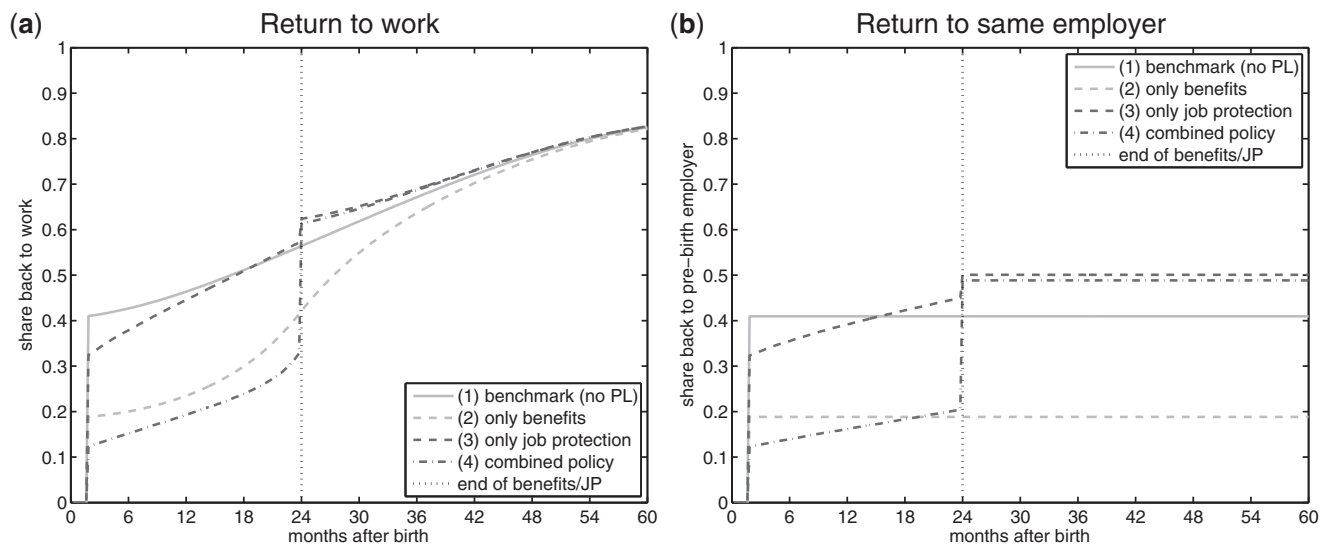

FIGURE 10

Alternative return-to-work profiles.

Notes: The figure shows return-to-work (a) and return-to-same-employer profiles (b) under one factual and three counterfactual policy regimes: (1) benchmark regime with no PL after job protected and paid maternity leave of 2 months, (2) benefits during 24 months but no job protection, (3) job protection during 24 months but no benefits. The fourth profile shows the combined policy (factual) with 24 months of benefits and job protection. The figures depict expected return to work profiles of a random sample of 1000 mothers from the estimation sample (giving birth between 1996 and 2000). For each mother in the sample, we fix 25 draws from the home production distribution. Then we calculate reservation wages, hazards and, finally, the aggregate failure function for the different policy configurations.

Source: Own calculations, based on estimates of the structural model

benefits achieve these aims 4 We simulate return to work profiles in three counterfactual PL systems (Figure 10, Table 6). In all systems, PL begins after 2 months of mandatory maternity leave which is fully paid and job protected. The benchmark counterfactual system assumes that there is no PL after the maternity leave period. The second system pays PL benefits until the second birthday of the child but there is no guarantee to return to the previous job. The third system guarantees the option to return to the pre-birth employer until the child turns 2 years old but does not provide benefits. We contrast the return-to-work profiles in these counterfactual systems with the factual system that offers a combination of both PL benefits and job protection until the child turns 2 years.

Consider first the benchmark case with no PL provisions after the end of maternity leave. In this system, about $41 \%$ of all women return to work immediately after maternity leave ends (Figure 10 and row 1 of Table 6). These women continue working for the pre-birth employer (Figure 10b). The remaining women re-enter the labour market looking for a new job. The simulations suggest that 24 months after birth around 56\% have returned to work, and 60 months after birth about $82 \%$ of all women have ever returned to work.

A system that pays PL benefits but grants no job protection delays return to work for two reasons. The share of women who return to the same employer is smaller. Whereas $41 \%$ return to

41. We abstract from a number of additional issues that are central for a comprehensive discussion of PL. For instance, our discussion does not quantify the budgetary incidence of the different systems, neither do we assess the costs on employers incurred due to job protection nor from the role of maternal care for child development. These issues are clearly important for a comprehensive assessment of PL policies. Nevertheless, we regard knowledge on the role of benefits and job protection for return to work decisions to be of first-order importance and focus on providing this evidence. 
TABLE 6

Return to work in alternative policy regimes

\begin{tabular}{|c|c|c|c|c|c|c|c|}
\hline \multirow[b]{2}{*}{ Percent back to work after (months) } & \multicolumn{3}{|c|}{ During leave (months) } & \multicolumn{4}{|c|}{ After leave (months) } \\
\hline & 2 & 12 & 23 & 24 & 36 & 48 & 60 \\
\hline $\begin{array}{l}\text { Benchmark } \\
\text { 1. No job protection/benefits }\end{array}$ & 41 & 46 & 56 & 56 & 67 & 76 & 82 \\
\hline $\begin{array}{l}\text { Deviation from benchmark } \\
\text { 2. Only } 24 \text { months benefits } \\
\text { 3. Only } 24 \text { months job protection } \\
\text { 4. } 24 \text { months job protection, } 24 \text { months benefits }\end{array}$ & $\begin{array}{r}-22 \\
-9 \\
-29\end{array}$ & $\begin{array}{r}-23 \\
-1 \\
-27\end{array}$ & $\begin{array}{r}-16 \\
0 \\
-25\end{array}$ & $\begin{array}{r}-14 \\
6 \\
5\end{array}$ & $\begin{array}{r}-3 \\
2 \\
2\end{array}$ & $\begin{array}{r}-1 \\
1 \\
1\end{array}$ & $\begin{array}{l}0 \\
1 \\
1\end{array}$ \\
\hline 5. Sum of rows 2 and 3 & -31 & -24 & -16 & -8 & -1 & 0 & 1 \\
\hline
\end{tabular}

Notes: This table reports how many women have returned to work in the benchmark regime (row 1 , only maternity insurance, cf. Figure 10, and the deviations from benchmark in the other regimes. Row (2) presents the system with 24 months of PL benefits but no job protection. Row (3) introduces 24 months of job protection without benefits. Row (5) calculates the sum of rows (2)-(3). Row (4) has 24 months of both job protection and benefits. Regimes in (1) to (3) are counterfactual, the regime in (4) is the Austrian regime between July 1990 and June 1995.

Source: Own calculations, based on estimates of the structural model

the same employer in the benchmark, only $19 \%$ return to the same employer immediately after the end of maternity leave because benefits increase the reservation wage (Figure 10p and row 2 of Table 6. Moreover, those who re-enter the labour market by looking for a new job return to work at a slower rate during the time when PL benefits are still paid (until month 24) than when PL benefits have run out (month 25 onwards). There is no spike at PL benefit exhaustion since the reservation wage adjusts smoothly with forward-looking agents. Eventually, 16 percentage points less women have returned by month 24 but the difference to the benchmark case with neither benefits nor job protection vanishes by month 60 . The benefits-only system generates more time with the child immediately after birth but it reduces the share returning to the same employer during the first 24 months. Moreover, a higher proportion of women stays out of the labour market even after 24 months. This is because the benefits-only system delays return to work not only in the first 2 years (as intended). This shows up also in the 3rd and 4th year after birth of the child since it takes time until sufficiently attractive job offers induce women still at home to go back to work.

A system that offers job protection without benefits delays return to work only slightly, on average (Figure 10b and row 3 of Table 6 . Immediately after maternity leave, 9 pctp. less women return to their pre-birth jobs compared to the benchmark. The share having returned to work very quickly converges to the baseline share during the first 18 months. Interestingly, the proportion of women taking up their pre-birth job remains considerably higher with job protection than without it. Whereas about $41 \%$ of all women return to the pre-birth employer in the baseline regime, the share is 9 pctp. higher in the system with job protection. The system with job protection therefore generates some time with the newborn child, maintains medium-run labour market participation, and increases the number of women who can continue their pre-birth job.

The system that offers a combination of both benefits and job protection delays return to work substantially in comparison with the benchmark (Figure 10b and row 5 of Table 6). The share that returns to pre-birth jobs initially is 29 pctp. lower. In month 23 , just before benefits and job protection end, still 25 pctp. less women have returned. In contrast, when benefits and job protection end, the share having returned is somewhat higher (5 pctp.) than in the benchmark situation without benefits nor job protection. Five years after giving birth to their child, $83 \%$ of women have returned to work in this generous system, even slightly more than in the benchmark 
with no PL (82\%). In all, $49 \%$ of women return to the same employer compared to $41 \%$ in the benchmark, a substantial difference.

Clearly, the combined system generates more time for care immediately after birth and higher medium-run employment compared to a system that pays only cash benefits or grants only job protection. Consider row 5 of Table 6 where we sum the deviations from benchmark of the two isolated policies. This illustrates the importance of interaction effects between the two policy instruments. The combined policy exceeds the sum of applying both instruments in achieving the twin goals of parental leave. The share still on leave in the combined regime relative to the sum of the isolated regimes is 3 pctp. higher after 12 months and 9 pctp. higher after 23 months, while the share back to work is 13 pctp. higher after 2 years and stays higher until 5 years after birth. This finding shows that cash benefits and job protection complement each other in achieving time for care immediately after birth while maintaining medium run labour market attachment.

\section{CONCLUSIONS}

This article studies the causal effect of alternative PL systems on short- and medium-run labour market outcomes of mothers by analysing three major changes to PL regulations in Austria. The contribution of the article is 2-fold. On the one hand, we provide reduced-form evidence of the causal effect of alternative PL systems on return-to-work, job continuity (return-to-sameemployer) as well as on employment and earnings in the medium run. On the other hand, we set up and structurally estimate a behavioural (non-stationary search) framework that sheds new light on the respective impact of the two main PL policy instruments: cash benefits and job protection. Within this framework, we can study how the two policy parameters affect return-to-work and return-to-same-employer behaviour, both in the short run and in the medium run.

The reduced-form evaluation exercise reveals that longer PL durations induce a significant delay in return-to-work. Extending both cash benefits and job protection by 1 year (the 1990 reform) increases the time at home after birth by 7.8 months. Reducing the duration of benefit payments by 6 months while keeping job protection at 24 months (the 1996 reform) shortens time at home by 3.4 months. Finally, extending payment duration by 12 months again keeping job protection at 24 months (the 2000 reform) extends time at home by 3.0 months. We also show that these changes are driven by both delays in return to pre-birth employer and delays in return to new jobs. Despite the significant delays in return-to-work among mothers exposed to the more generous leave regimes, we find no detrimental effects on their labour market outcomes in the medium-run.

The structural model suggests that introducing deterministic PL durations into a search model helps us better understand the role of cash benefits and job protection duration in PL policies. Our non-stationary job-search framework features a changing mean of the wage offer distribution; a changing value of home production over time; and an outside option (return-to-same-employer) which is time-delimited. Using the estimates obtained from this novel structural framework, we conduct out-of-sample predictions. We find that our model predicts return-to-work behaviour and return-to-same-employer behaviour remarkably well, both in the short-run and in the medium-run.

Structural estimates of the model of job search suggest that mothers value time with their child more strongly right after birth than later on in their child's life. Moreover, the time period we analyse is one where there is very little child care for children below the age of 3 years. In this setting, a PL policy can support families of newborn children by generating opportunities for prolonged parental care immediately after birth while maintaining medium-run labour market attachment of parents. We simulate return-to-work behaviour under counterfactual policies and measure how much time for care and medium-run employment these systems generate. We find that the system that combines both policy instruments generates more care immediately after birth 
and more employment in the medium run than systems that just use one or neither of the policy instruments. We conclude that the the two PL policy instruments need to be jointly implemented in order to achieve both goals. They interact to subsidize time for parental care immediately after birth while maintaining medium-run labour market attachment.

\section{A. APPENDIX}

\section{A.1. Investigating selection into employment}

We examine differential selection into employment in Figure A.1 panels a, b, and c for the three policy reforms. The first quadrant in each of the three panels shows $D I D-R D$ impacts of the reforms on employment rates (along with confidence intervals) by year since the child's birth. As expected, in the second year after childbirth, employment rates of mothers in the less generous regimes are higher compared to those of mothers in the more generous regimes. Nevertheless, there are no differences in employment rates between pre- and post-July mothers starting from year 3 after child's birth when both groups have exhausted their respective PL provisions. Interestingly, despite the fact that mothers giving birth in the more generous regimes were less likely to have ever returned to work (as seen in Section 4.2], employment rates of mothers in the more and less generous regimes are virtually identical starting from year 3 after birth. The contrasting result in these two outcomes is explained by the fact that a larger share of mothers in the less generous regimes returned to work but only for a short period of time.

The following set of figures in panels a,b, and c of Figure A.1 check for differential selection into employment in each of the years following childbirth by comparing pre-birth labour market outcomes of pre- and post-July mothers in the reform year relative to mothers in the pre-reform year by employment status. In year 2 after birth, we observe that employed mothers who gave birth in the more generous leave regimes are positively selected (i.e., they have better pre-birth labour market outcomes relative to mothers employed in the less generous regimes). Starting from year 3 , once employment rates of pre- and post-July mothers equalize, we see no further evidence of differential selection into employment.

\section{A.2. Likelihood contributions}

Assume that individual-specific home production values follow the distribution $c \sim G(c)$. To derive the likelihood contribution of individual $i$, note that there are three possible outcomes.

(1) $i$ is still searching in $T$ (the end of the observation period).

(2) $i$ returned to her pre-birth job in $t_{i}$.

(3) $i$ found a new job in $t_{i}$.

Given the parameters of the model, the optimal time of returning to the pre-birth job is uniquely determined by $c$. This follows from the fact that the reservation wage at any point in time is increasing in $c$, i.e. the higher home production, the later mothers return to their pre-birth jobs. Given a continuous distribution for $c$, it is thus possible to derive the bounds on $c$ such that return in $t$ is certain (unless a valuable job offer arrives before $t$. 42 .

Let $\underline{c}(t)$ denote the lowest possible $c$ such that the mother returns in $t$ but not earlier and let $\bar{c}(t)$ denote the highest possible $c$ such that the mother returns in $t$ but not later. Then all $c \in[\underline{c}(t), \bar{c}(t)]$ return to their pre-birth jobs in $t$.

The likelihood of outcome (1), mothers still on PL in $T$, is jointly given by (a) the probability that $i$ does not return to her pre-birth job at or before $\tau_{o}$, the end of job protection, and (b) the probability that $i$ did not find a suitable job offer. The probability of not returning in $\tau_{o}$ or before is given by

$$
P(\text { no return to } \mathrm{pb} \text { job })=P\left(c_{i}>\bar{c}\left(\tau_{o}\right)\right)=1-G\left(\bar{c}\left(\tau_{o}\right)\right)
$$

and the probability of not receiving a sufficiently high job offer in any $t \leq T$ is given by

$$
P(\text { still searching in } T)=\int_{\bar{c}\left(\tau_{o}\right)}^{\infty} \prod_{j=0}^{T}\left[\left(\lambda \Phi\left(\frac{\eta_{i j}}{\sigma_{w}}\right)+(1-\lambda)\right)\right] d G(c)
$$

where $\eta_{j}=\log \left(w_{j}^{*}\right)-\mu_{w}$. This expression integrates the probability of receiving and rejecting a wage offer in any period (wage offers are lognormal, $w \sim \log \mathrm{N}\left(\mu_{w}, \sigma_{w}\right)$ ) and the probability of not receiving an offer over all home production values which do not return to their pre-birth jobs (then the probability of observing them still searching would be zero).

42. Uniqueness of the optimal time of return requires decreasing reservation wages, as proven in Friiters and Van der Klaauw 2006). We check for this condition at the solution. 

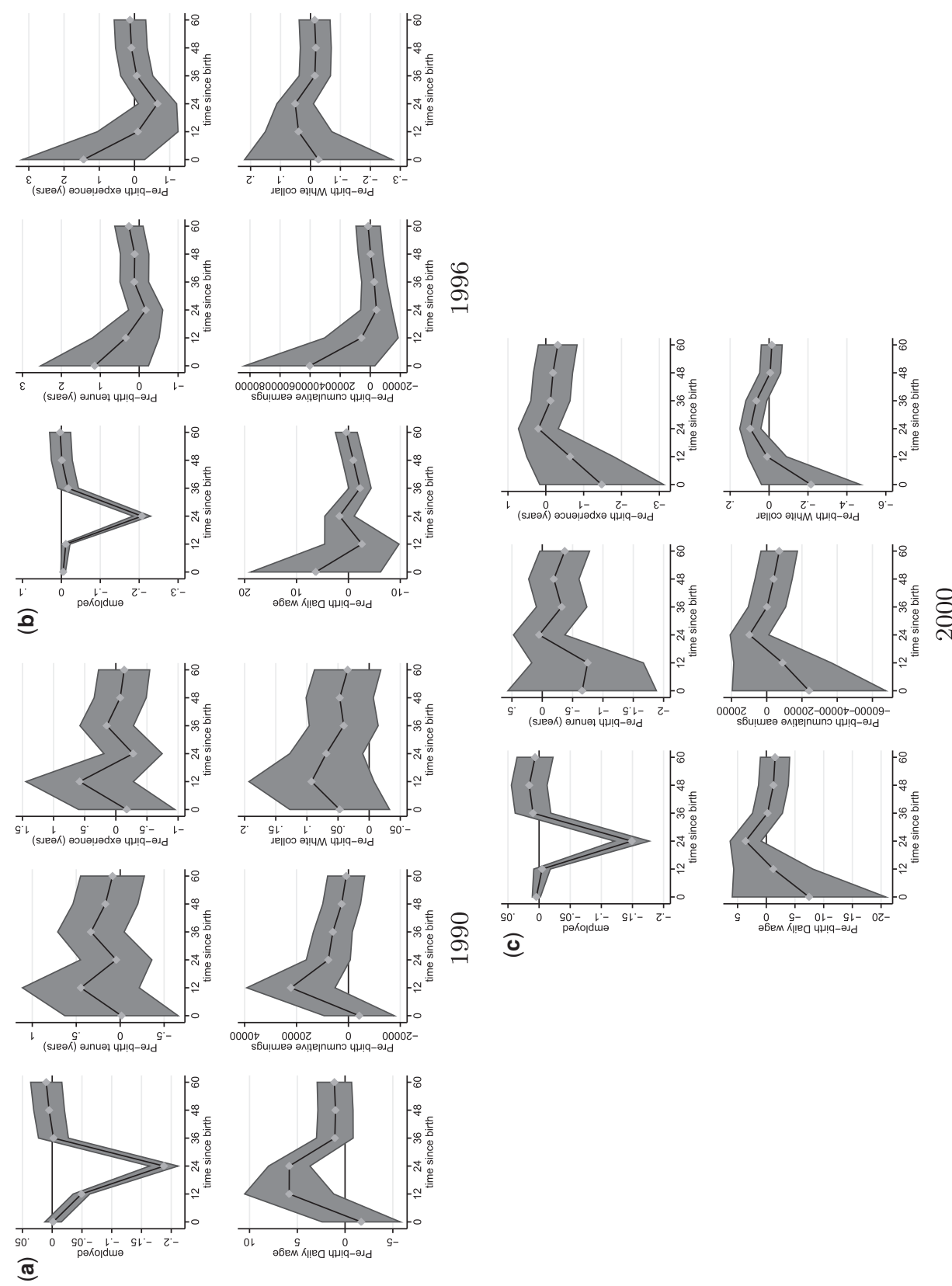
The full likelihood contribution of an observation still on leave is thus

$$
\left[1-G\left(\bar{c}\left(\tau_{o}\right)\right)\right] \int_{\bar{c}\left(\tau_{o}\right)}^{\infty} \prod_{j=0}^{T}\left[\left(\lambda \Phi\left(\frac{\eta_{i j}}{\sigma_{w}}\right)+(1-\lambda)\right)\right] d G(c) .
$$

The likelihood of outcome (2), mothers who returned to their pre-birth jobs in $t_{i}$, is jointly determined by the probability of return in $t_{i}$ and the probability of not receiving and accepting a superior job offer at any $t \in\left[0, t_{i}\right]$. The former is given by

$$
P\left(\text { return to pb job in } t_{i}\right)=G\left(\bar{c}\left(t_{i}\right)\right)-G\left(\underline{\mathrm{c}}\left(t_{i}\right)\right)
$$

while the latter is given by

$$
P\left(\text { still searching in } t_{i}\right)=\int_{\underline{c}\left(t_{i}\right)}^{\bar{c}\left(t_{i}\right)} \prod_{j=0}^{t_{i}}\left[\left(\lambda \Phi\left(\frac{\eta_{i j}}{\sigma_{w}}\right)+(1-\lambda)\right)\right] d G(c) .
$$

Finally, the likelihood of outcome (3), mothers who accepted a new job in $t_{i}$, is jointly determined by the probability of not returning at any $t<t_{i}$ (return in $t_{i}$ is possible, since new wage offers are accepted at the beginning of the period), the probability of not receiving and accepting a good wage offer before $t_{i}$ and the probability of receiving and accepting offer $\hat{w}$ in $t_{i}$. The former two are analogues to the cases above, while the probability of accepting and observing $\hat{w}$ is more involved.

Note that in all cases so far the likelihoods involved the true unknown wage-offer reservation wage. The probability of $\hat{w}$, however, involves the joint (normal) distribution of wage offers and measurement error. Let $\xi=\epsilon+u$ be the observed residual given wage offer $\hat{w}_{i}$ and $\mu_{i, t}$. Then, $u$ and $\xi$ follow a joint normal distribution with

$$
\begin{aligned}
\sigma_{\xi} & =\sqrt{\sigma_{w}^{2}+\sigma_{u}^{2}} \\
\rho & =\frac{\sigma_{w}}{\sigma_{\xi}} .
\end{aligned}
$$

Thus, the joint probability of receiving and accepting offer $\hat{w}$ is given by

$$
P\left(\text { exit in } t_{i} \text { and observe } \hat{w}\right)=\lambda \Phi\left(\frac{\rho / \sigma_{\xi}\left(\log \hat{w}-\mu_{w}\right)-\eta_{i t_{i}}}{\sqrt{1-\rho^{2}}}\right) \frac{1}{\hat{w}} \phi\left(\frac{\log \hat{w}-\mu_{w}}{\sigma_{\xi}}\right) \frac{1}{\sigma_{\xi}} .
$$

This expression is derived and discussed in more detail in Wolpin 1987). The full likelihood contribution of a mother accepting a new job in $t_{i}$ with observed wage $\hat{w}$ is then

$$
\begin{aligned}
& {\left[1-G\left(\underline{c}\left(t_{i}\right)\right)\right] } \int_{\underline{\mathbf{c}}\left(t_{i}\right)}^{\infty}\left\{\prod_{j=0}^{t_{i}-1}\left[\lambda \Phi\left(\frac{\eta_{i j}}{\sigma_{w}}\right)+(1-\lambda)\right]\right. \\
&\left.\lambda \Phi\left(\frac{\rho / \sigma_{\xi}\left(\log \hat{w}-\mu_{w}\right)-\eta_{i t_{i}}}{\sqrt{1-\rho^{2}}}\right) \frac{1}{\hat{w}} \phi\left(\frac{\log \hat{w}-\mu_{w}}{\sigma_{\xi}}\right) \frac{1}{\sigma_{\xi}}\right\} d G(c) .
\end{aligned}
$$

In the estimation, the integrals are approximated by simulation methods. We fix 25 draws from the Uniform distribution for every observation in the sample prior to the estimation. In every estimation round, these draws are converted to draws from the truncated home production distribution and then the whole paths of reservation wages computed. There is bias in the parameter estimates from simulated maximum likelihood, but according to simulations in Börsch-Supan and Hajivassiliou 1993) the bias tends to zero if the number of draws exceeds 20. 


\section{A.3. Age profiles of individual-specific parameters}

(a) Offer probability $\lambda$

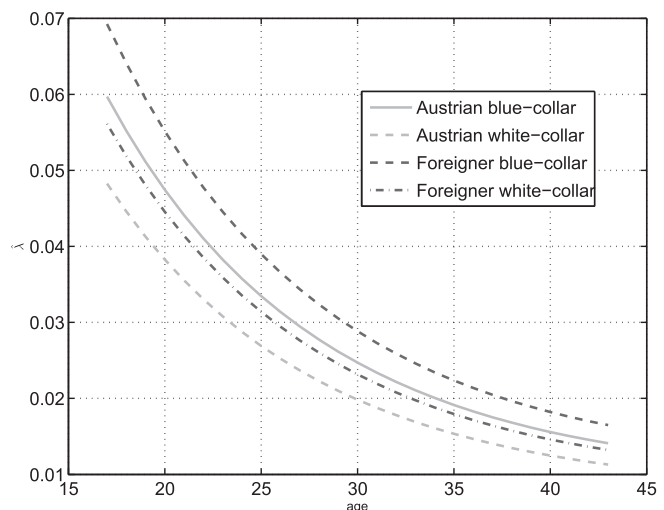

(b) Home production $\mu_{c}$

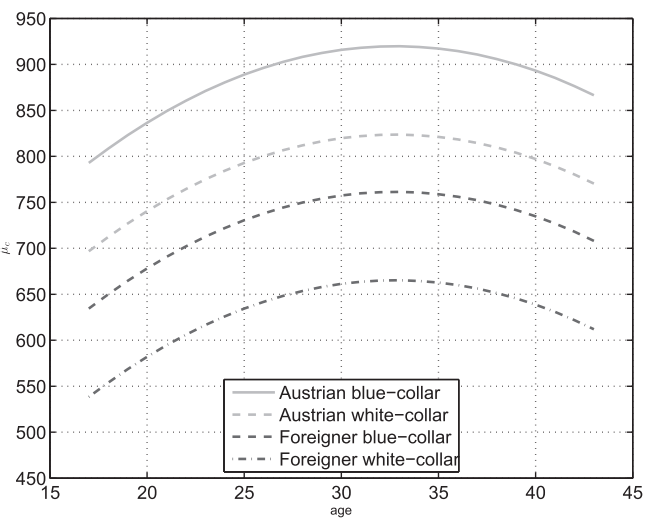

(c) Median log wage offer

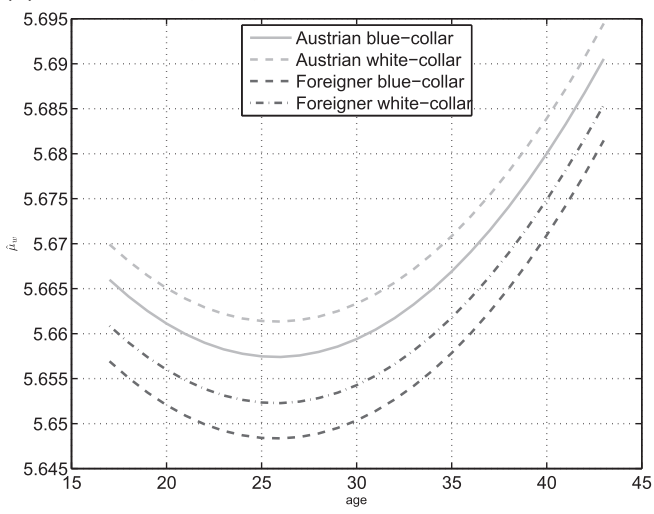

FiguRE A.2

Age profiles for results of Table 5

Notes: Figures plot predicted $\lambda, \mu_{c}$, and $\mu_{w}$ for mothers working in manufactoring in West Austria (pre-birth), with median pre-birth wage (287 euro/week) 


\section{A.4. Return to work with no extra child}

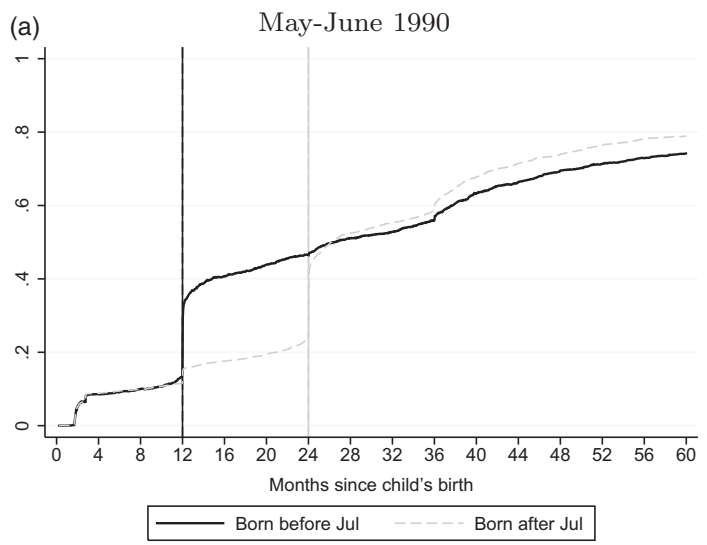

(b) July-August 1996
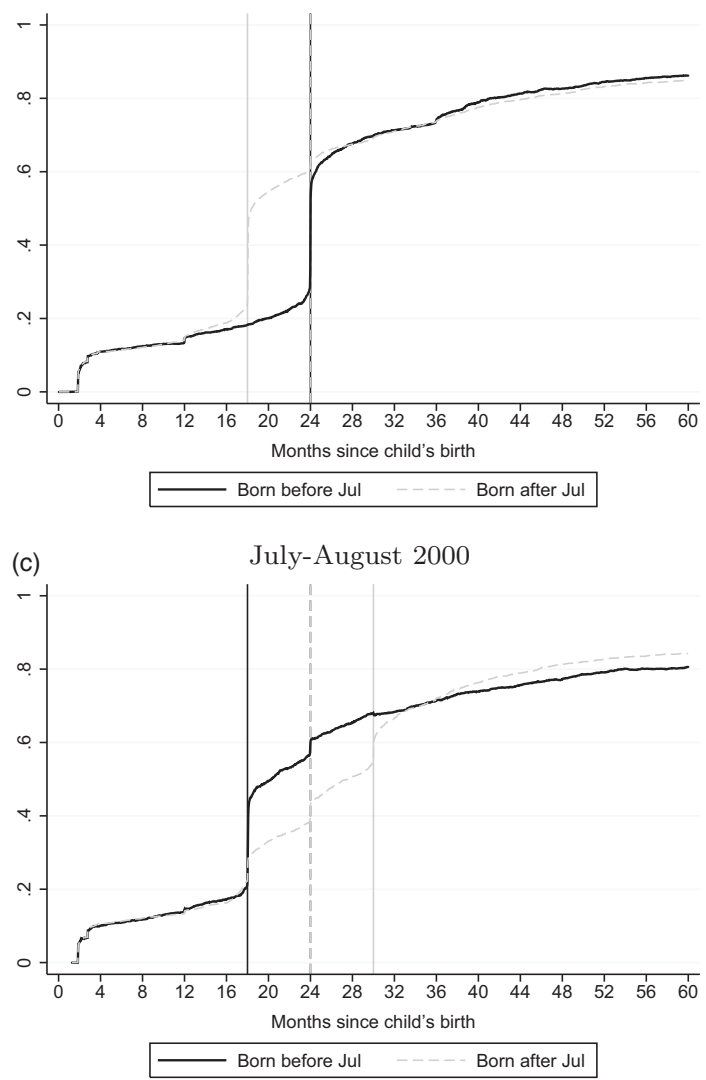

FIGURE A.3

Return to work-failure functions-Parity 1 No Extra Kids.

Notes: This figure shows the proportion who have returned to work at or before $t$ months after child's birth. The sample includes mothers giving birth at parity one between May 1st and August 30th of 1990 (panel a), 1996 (panel b), and 


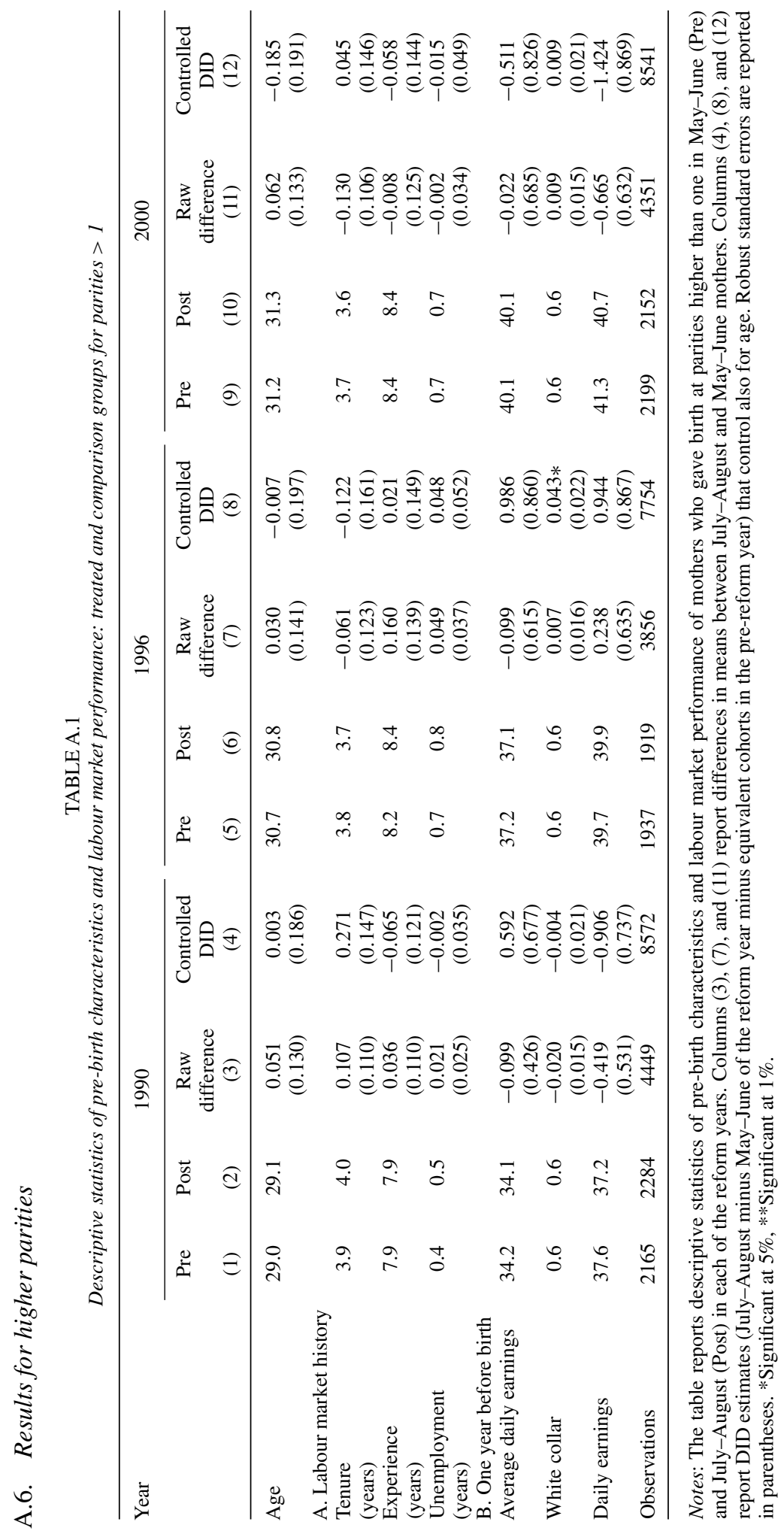


LALIVE ET AL. PARENTAL LEAVE AND MOTHERS' CAREERS

TABLE A.2

The causal effects of the reforms for mothers giving birth at parities $>1$

\begin{tabular}{lccc}
\hline Year of reform & 1990 & 1996 & 2000 \\
& $(1)$ & $(2)$ & $(3)$ \\
\hline Time at home (censored at 60 months) & $5.099^{* *}$ & $3.887^{* *}$ & $4.901^{* *}$ \\
& $(0.873)$ & $(0.841)$ & $(0.756)$ \\
& 27.920 & 26.821 & 25.793 \\
Time until return to same employer (for those back to pre-birth job) & $6.515^{* *}$ & $3.073^{* *}$ & $3.073^{* *}$ \\
& $(0.655)$ & $(0.708)$ & $(0.590)$ \\
Time until return to new employer (for those back to new job) & 12.206 & 16.693 & 15.788 \\
& 2.330 & $2.718^{*}$ & $5.181^{* *}$ \\
Back within 60 months & $(1.247)$ & $(1.164)$ & $(1.079)$ \\
& 29.556 & 29.084 & 28.727 \\
Back to pre-birth employer (censored at 60 months) & -0.031 & -0.032 & $-0.04^{*}$ \\
& $(0.018)$ & $(0.018)$ & $(0.016)$ \\
& 0.770 & 0.845 & 0.857 \\
Daily wage at 1st job after birth & -0.026 & $-0.047^{*}$ & -0.036 \\
& $(0.020)$ & $(0.023)$ & $(0.020)$ \\
Daily wage at 1st job after birth (pre-birth employer) & 0.498 & 0.570 & 0.573 \\
& 1.012 & $-1.727^{*}$ & -0.402 \\
& $(0.760)$ & $(0.866)$ & $(0.829)$ \\
Daily wage at 1st job after birth (new employer) & 31.700 & 34.242 & 31.073 \\
& 0.432 & $-2.855^{* *}$ & -0.843 \\
Number of observations & $(0.912)$ & $(1.032)$ & $(1.045)$ \\
& 33.713 & 36.059 & 31.617 \\
& 2.124 & 0.088 & -0.454 \\
& $(1.314)$ & $(1.518)$ & $(1.326)$ \\
& 28.019 & 30.470 & 29.973 \\
& 8572 & 7754 & 8541 \\
\end{tabular}

Notes: This table reports DID-RD estimates for the impacts of the 1990, 1996, and 2000 reforms. The samples include all mothers who gave birth at parities higher than one between May 1st and August 30th. Regressions compare differences in outcomes between the cohort exposed to the more generous regime and the cohort exposed to the less generous regime relative to the outcomes of pre- and post-July mothers who gave birth in the year preceding the reform. Estimates come from regressions that control for age at birth, and the following indicators for mothers, labour market performance measured 12 months before the child's birth: tenure, experience, months of unemployment, cumulative income, daily wages, and indicators for industry, region, and white-collar occupation. Regressions also control for the unemployment rates in the region of pre-birth employment at the end of the job protection and benefits payments periods. Robust standard errors are reported in parentheses. Means of the comparison group (i.e., the group with access to the less generous regime) are reported in italics. *Significant at $5 \%, * *$ Significant at $1 \%$.

TABLE A.3

The causal effects of the reforms on labour market outcomes in year 5 for parities $>1$

\begin{tabular}{|c|c|c|c|}
\hline Year of reform & $\begin{array}{c}1990 \\
(1)\end{array}$ & $\begin{array}{c}1996 \\
(2)\end{array}$ & $\begin{array}{c}2000 \\
(3)\end{array}$ \\
\hline \multicolumn{4}{|c|}{ A. Cumulative Outcomes in Year 5} \\
\hline Months in employment & $\begin{array}{c}-2.792^{* *} \\
(0.790) \\
22.219\end{array}$ & $\begin{array}{c}-3.256^{* *} \\
(0.769) \\
25.447\end{array}$ & $\begin{array}{c}-3.908^{* *} \\
(0.720) \\
25.688\end{array}$ \\
\hline Months unemployed & $\begin{array}{c}-3.037^{* *} \\
(0.451) \\
7.155\end{array}$ & $\begin{array}{c}-0.771^{*} \\
(0.306) \\
3.951\end{array}$ & $\begin{array}{c}-0.696^{*} \\
(0.279) \\
3.637\end{array}$ \\
\hline Cumulative earned income & $\begin{array}{c}-2887^{*} \\
(1255.2) \\
31,850\end{array}$ & $\begin{array}{c}-3477^{*} \\
(1427.3) \\
39,034\end{array}$ & $\begin{array}{c}-4833^{* *} \\
(1434.3) \\
40,174\end{array}$ \\
\hline
\end{tabular}

(Continued) 
TABLE A.3

Continued

\begin{tabular}{|c|c|c|c|}
\hline Year of reform & $\begin{array}{c}1990 \\
\text { (1) }\end{array}$ & $\begin{array}{c}1996 \\
(2)\end{array}$ & $\begin{array}{c}2000 \\
(3)\end{array}$ \\
\hline \multicolumn{4}{|c|}{ B. Labour Market Outcomes in Year 5} \\
\hline \multirow[t]{3}{*}{ Employed } & -0.001 & 0.000 & -0.036 \\
\hline & $(0.021)$ & $(0.023)$ & $(0.021)$ \\
\hline & 0.548 & 0.660 & 0.667 \\
\hline \multirow[t]{3}{*}{ Working for pre-birth firm } & 0.013 & -0.032 & 0.019 \\
\hline & $(0.028)$ & $(0.028)$ & $(0.026)$ \\
\hline & 0.519 & 0.519 & 0.465 \\
\hline \multirow[t]{3}{*}{ Tenure with current employer } & -0.863 & -4.695 & 0.215 \\
\hline & $(2.576)$ & $(2.542)$ & $(2.355)$ \\
\hline & 67.401 & 65.105 & 58.823 \\
\hline \multirow[t]{3}{*}{ Months worked } & 0.016 & -0.142 & $-0.507^{*}$ \\
\hline & $(0.236)$ & $(0.250)$ & $(0.227)$ \\
\hline & 6.607 & 7.898 & 8.038 \\
\hline \multirow[t]{3}{*}{ Earnings per day worked } & 1.213 & -0.958 & -0.694 \\
\hline & $(0.969)$ & (1.014) & $(0.894)$ \\
\hline & 41.785 & 44.010 & 44.345 \\
\hline \multirow[t]{3}{*}{ Earnings per calendar day } & 0.356 & -0.655 & -2.002 \\
\hline & (1.018) & $(1.175)$ & $(1.051)$ \\
\hline & 22.909 & 29.057 & 29.362 \\
\hline \multirow[t]{3}{*}{ Annual income } & 66.5 & 477.7 & $-1,294^{* *}$ \\
\hline & $(373.3)$ & $(479.1)$ & (411.3) \\
\hline & 9414 & 11912 & 12031 \\
\hline Number of observations & 8572 & 7754 & 8541 \\
\hline
\end{tabular}

Notes: This table reports DID-RD estimates for the impacts of the 1990, 1996, and 2000 reforms. The samples include all mothers who gave birth at parities higher than one between May 1st and August 30th. Regressions compare differences in outcomes between the cohort exposed to the more generous regime and the cohort exposed to the less generous regime relative to the outcomes of pre- and post-July mothers who gave birth in the year preceding the reform. Estimates come from regressions that control for age at birth, and the following indicators for mothers, labour market performance measured 12 months before the child's birth: tenure, experience, months of unemployment, cumulative income, daily wages, and indicators for industry, region, and white-collar occupation. Regressions also control for the unemployment rates in the region of pre-birth employment at the end of the job protection and benefits payments periods. Robust standard errors are reported in parentheses. Means of the comparison group (i.e. the group with access to the less generous regime) are reported in italics. *Significant at $5 \%, * *$ Significant at $1 \%$.

Acknowledgments. We would like to thank three anonymous referees and the editor for helpful comments on a previous version of the article. We have also benefitted from discussions with Marnix Amand, Gerard van den Berg, David Blau, Thomas Piketty, Emmanuel Saez, Betsey Stevenson, Justin Wolfers, participants at the IFN workshop on family policy in Stockholm, the ESSLE conference in Buch/Ammersee, the 10th World Congress of the Econometric Society, and the ZEW microsimulation conference in Mannheim, and seminar participants at the Federal Reserve Bank of Chicago, IFAU, Stockholm University, Hebrew University, University of Hannover, and the University of Linz. The research was funded by the Austrian Science Fund (FWF): S 10304-G16. Rafael Lalive acknowledges financial support from the Swiss National Center of Competence in Research LIVES. Andreas Steinhauer acknowledges financial support from the Swiss National Science Foundation.

\section{Supplementary Data}

Supplementary data are available at Review of Economic Studies online.

\section{REFERENCES}

BAKER, M. and MILLIGAN, K. (2005), "How Does Job-Protected Maternity Leave Affect Mothers' Employment and Infant Health?" (Working Paper No. 11135, NBER).

BAUM, C.L. (2003), "The Effect of State Maternity Leave Legislation and the 1993 Family and Medical Leave Act on Employment and Wages", Labor Economics, 10, 573-596.

BÖRSCH-SUPAN, A. and HAJIVASSILIOU, V.A. (1993), "Smooth Unbiased Multivariate Probability Simulators for Maximum Likelihood Estimation of Limited Dependent Variable Models", Journal of Econometrics, 58, 347-368. 


\section{LALIVE ET AL. PARENTAL LEAVE AND MOTHERS' CAREERS}

BRUNNER, B. and KUHN, A. (2011), “Financial Incentives, the Timing of Births, Birth Complications, and Newborns' Health: Evidence from the Abolition of Austria's Baby Bonus" (Discussion Paper No. 6141, IZA).

EJRNAES, M. and KUNZE, A. (2006), "What is Driving the Family Gap in Women's Wages?" Technical report, mimeo, Norwegian School of Economics and Business, Bergen, Norway.

FLINN, C. and HECKMAN, J.J. (1982), "New Methods for Analyzing Structural Models of Labor Force Dynamics", Journal of Econometrics, 18, 115-168.

FRIJTERS, P. and VAN dER KLAAUW, B. (2006), "Job Search with Nonparticipation", The Economic Journal, 116, 45-83.

GOULD, E.D. and SIMHON, A. (2011), "Does Quality Time Produce Quality Children? Evidence on the Intergenerational Transmission of Human Capital Using Parental Deaths" (Discussion Paper No. 8258, CEPR).

GURYAN, J., HURST, E. and KEARNEY, M. (2008), "Parental Education and Parental Time with Children", The Journal of Economic Perspectives, 22, 23-46.

HAHN, J., TODD, P. and VAN DER KLAAUW, W. (2001), "Identification and Estimation of Treatment Effects with a Regression-Discontinuity Design", Econometrica, 69, 201-209.

HASHIMOTO, M., PERCY, R., SCHOELLNER, T. and WEINBERG, B.A. (2004), "The Long and Short of it: Maternity Leave Coverage and Women's Labor Market Outcomes". (Discussion Paper No. 1207, IZA).

KLERMAN, J.A. and LEIBOWITZ, A. (1997), "Labor Supply Effects of State Maternity Leave Legislation", in Blau, F. and Ehrenberg, R. (eds) Gender and Family Issues in the Workplace (New York: Russell Sage Press) 65-85.

KLERMAN, J.A. and LEIBOWITZ, A. (1999), "Job Continuity Among New Mothers", Demography, 36, 145-155.

LALIVE, R. and ZWEIMÜLLER, J. (2009), "How Does Parental Leave Affect Fertility and Return to Work? Evidence from Two Natural Experiments", The Quarterly Journal of Economics, 124, 1363-1402.

OECD. Employment outlook, 1993, 2001, 2004.

RUHM, C.J. (1998), “The Economic Consequences of Parental Leave Mandates: Lessons from Europe”, The Quarterly Journal of Economics, 113, 285-317.

SCHÖNBERG, U. and LUDSTECK, J. (2007), Maternity Leave Legislation, Female Labor Supply, and the Family Wage Gap" (Discussion Paper No. 2699, IZA).

VAN DEN BERG, G.J. (1990), "Nonstationarity in Job Search Theory”, The Review of Economic Studies, 57, $255-277$.

WALDFOGEL, J. (1999), The Impact of the Family and Medical Leave Act”, Journal of Policy Analysis and Management, 18, 281-302.

WOLPIN, K.I. (1987), Estimating a Structural Search Model: The Transition from School to Work", Econometrica, 55, 801-817. 